\title{
Referential Revealed Preference Theory
}

\author{
Hassan Nosratabadi* \\ This Draft: June 17, 2017
}

\begin{abstract}
Reference-dependent choice behavior implies behavioral anomalies such as the socalled attraction effect, status quo bias, and endowment effect. This paper builds a new theory of revealed preference capturing preferences that depend on a reference point. The first main contribution of this work is a decomposition of the Weak Axiom of Revealed Preference (WARP) into three independent axioms. Referential revealed preference theory is then, naturally, constructed by only removing the WARP-rationales that are inconsistent with the data. This minimal deviation, in addition to explaining the mentioned behavioral anomalies, preserves the predictive power of the classical theory to the extent possible. Therefore, all sensible results are endogenously derived from the model. These results include: formation of references and reference preferences, the connection of the latter concept to the classical revealed preference, the nature of referential effects, and the characterization of choice. Interestingly, the notion of sequential rationalizability arises, endogenously, as a result in the referential revealed preference theory.
\end{abstract}

JEL Classification: D11, D81.

Keywords: Reference-Dependent Choice, Reference Preferences, Attraction Effect, Status-Quo Bias.

\section{Introduction}

A theory, by its nature, asks for simplification of the complex reality. Such simplification is not merely meant to mitigate communication between the theorist and empiricist; it is a necessity through which falsifiable statements are generated. The justification of rational

* Department of Economics, Rutgers, the State University of New Jersey, 75 Hamilton Street, New Brunswick, NJ 08901-1248 (email: sn351@scarletmail.rutgers.edu). 
homo economicus is a sheer example of such consideration in the realm of social science. There is no doubt that a decision maker's (DM) choice is dependent on considerations that go beyond the scope of the rational paradigm. Among others, psychology of choice, in particular, is a neglected source. At the end, human brain, as the single unit in charge of a DM's behavior, is a complex system whose manner of functioning is still a question of significance in cognitive science and neuroscience.

Implicitly imposed in the cardinal notion of utility in the works of Marshall (1890) and Edgeworth (1881), and later in the ordinal concept of preferences by Pareto (1906), the notion of rationality first entered economic theory in a practical manner through the infamous revealed preference theory developed in Samuelson (1938). Though it has been shown to be equivalent to two preexisting approaches under a technical condition ${ }^{1}$, the revealed preference theory still serves a critical role in economic theory by bridging the gap between the pure theory of consumption and the observable choice behavior, hence guaranteeing the falsifiability of the former.

Essential to the revealed preference theory is its rational postulate: the weak axiom of revealed preference (WARP). The major prediction of the theory is summarized in the fundamental theorem of revealed preferences. Informally, the theorem states that, under WARP, the choice behavior of a DM's on any arbitrary bundle of alternatives completely submits to the (transitive) choice she makes on all possible pairs of elements in that bundle. ${ }^{2}$ Upon closer examination of the theorem, one can track the following rationales enforced upon the classical DM:

(i) Transitivity of pairwise choice

(ii) Independence of irrelevant alternatives

Figure 1 depicts the nature of this categorization. The first rationale, pairwise transitivity (PT), speaks to the fact that a WARP-rational DM acts transitive on doubletons. The second rationale asserts that the choice in any arbitrary bundle should be consistent with the choice in all pairs, making a third alternative irrelevant in the ranking of a pair. As depicted in Figure 1, this independence implies two directional rationalities: a top-down rationale (TDR) which requires the choice to be the most preferred element under pairwise comparison, and a bottom-up rational (BUR) which forces the best alternative from the perspective of pairwise choice to survive in the larger menu. WARP, therefore, guarantees existence of a preference, $\succsim^{p}$, often referred to as revealed preference, extracted from choice behavior on doubletons and rationalizing the choice; that is, the choice is made as if DM was maximizing $\succsim^{p}$.

\footnotetext{
${ }^{1}$ The condition is the continuity of the choice correspondence. For an elaboration on this see Arrow
} 


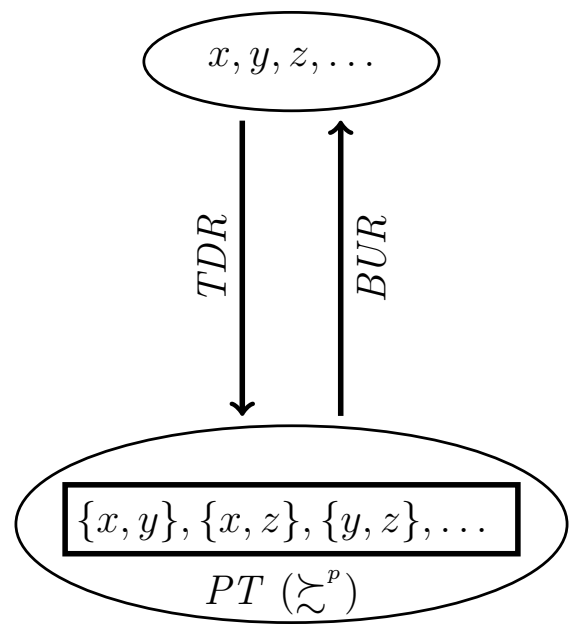

Figure 1: WARP Rationales

Experimental economists have taken advantage of the strong predictive power offered by WARP to test the classical revealed preference theory. These experimental works, as one expects, are mapped in a one-to-one manner to the categorization of rationales introduced in Figure 1; the first group tests the transitivity of pairwise choice; that is the cyclicality of choice, and the second speaks to the conformation to the pairwise choice; that is the so called menu effect. For the purpose of this paper, and following Ok, Ortovela, and Riella (2015), I concentrate on the latter phenomenon. ${ }^{3}$

Menu effect is further sub-branched based on a variety of behavioral considerations. Reference-dependent models play a key role in the contextualization of these effects. Originated in Kahneman and Tversky (1979) for risky environments, developed further in Kahneman and Tversky (1984), and extended to riskless environments in Tversky and Kahneman (1991), a reference-dependent model is, basically, characterized by a decision maker whose behavior is rationalized, as opposed to $\succsim^{p}$, by a family of reference preferences, $\succsim^{r}$, each of which operates from a reference point, $r$. As Tversky and Kahneman (1991) argue in detail, attraction effect ${ }^{4}$, status quo bias, and endowment effect could all be rationalized via loss aversion. To illustrate, consider Figure $23^{5}$ Attraction effect pertains to a break down of indifference when an asymmetrically dominated alternative is introduced into the menu. In Figure 2 , the indifference curves are drawn with respect to the revealed preference, $\succsim^{p}$. A risk averse

(1959), and Mas-Collel, Whinston, and Green (1995) chapters 1, and 3.

${ }^{2}$ This was first shown in Arrow (1959).

${ }^{3}$ This choice is supported by the evidence from Manzini and Mariotti (2010). Their experimental results show that the dominant factor of WARP anomalies are from the second category; that is the menu effect.

${ }^{4}$ This effect is also referred to as the asymmetrically dominated effect and decoy effect in the literature.

${ }^{5}$ The presentation offered in Figures 2, 3, and the essence of the argument that follows is borrowed from Tversky and Kahneman (1991). 
DM associates less risk in choosing $x$ than $y$ (both dominate $r$ ) when using $r$ as a reference point. This is because from $r$-point of view, $x$ only offers gain, but $y$ offers a trade-off: gain in Dimension 2 versus loss in Dimension 1. Denote the reference preference with respect to the reference point $r$ by $\succsim^{r}$. Then $x \sim^{p} y$ and $x \succ^{r} y$; that is $\succsim^{p}$ evolves to $\succsim^{r}$ by adapting to the reference point $r$. The same risk averse DM favors $y$ over $x$ when using $s$-point of view; that is $x \sim^{p} y$ but $y \succ^{s} x$. The experimental evidence for attraction effect is vast. See for example, Huber, Payne, and Puto (1982), Doyl, O'Conner, Reynolds, and Bottomly (1999), and Tversky and Kahneman (1991).

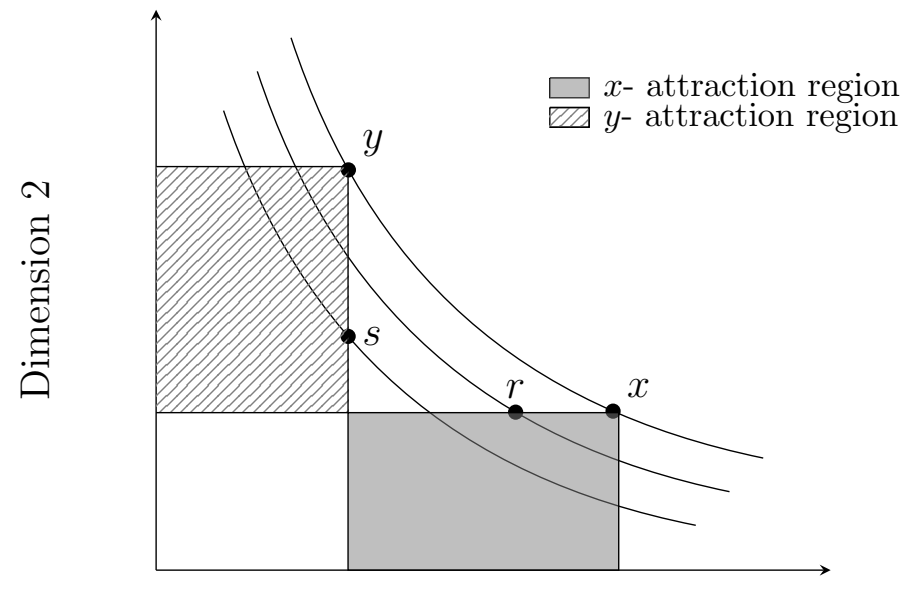

Dimension 1

Figure 2: Attraction Effect

The other major behavioral anomaly is the tendency to retain status quo. Assume that a DM, to start, is indifferent between alternatives $x$ and $y$. In period 1 , she is given one of these alternatives, and then makes a choice from the two in period 2. Figure 3 depicts the choice scenario in period 2. The main idea in status quo bias is that the initial endowments produce biases that break indifference. ${ }^{6}$ Assume that in period 1 the decision maker is given the alternative $x$. A risk averse DM who assigns higher weights to losses than gains will favor $x$ in period 2 to retain her status quo. Let $\succsim^{p}$ denote the preference derived from the pairwise comparison in period 1 (the one that matches the revealed preference theory) and $\left(a, t_{i}\right)$ denote the alternative $a$ in time $i$. Then $\left(x, t_{2}\right) \sim^{p}\left(y, t_{2}\right)$, but $\left(x, t_{2}\right) \succ^{\left(x, t_{1}\right)}\left(y, t_{2}\right)$. Obviously both $\left(x, t_{2}\right)$ and $\left(y, t_{2}\right)$ dominate $\left(x, t_{1}\right)$ in period 2 as the consumption in period 1 is not available anymore. The same argument works in the reverse direction when the

${ }^{6}$ This effect and its twin, endowment effect, are essentially the same. For a detailed discussion on this see Tversky and Kahneman (1991). We refer to this effect as the status-quo bias for the remainder of this paper. 
initial endowment is $y \cdot{ }^{7}$ The significance of this effect has also been vastly documented in the literature. See, for example, Samuelson and Zeckhauser (1988), Tversky and Kahneman (1991), Knetsch and Sinden (1984), and Knetsch (1989).

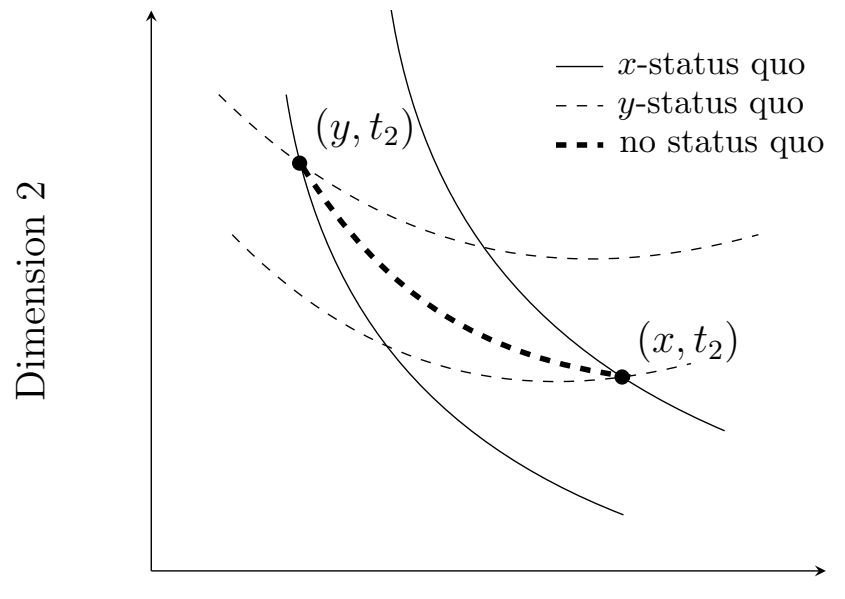

Dimension 1

Figure 3: Status-Que Bias (or Endowment Effect)

The reference dependent model developed in Tversky and Kahneman (1991) has been shown to have considerable significance in economic theory. ${ }^{8}$ The origin of reference preferences, however, is beyond the scope of these author's work. To this author's knowledge, this question has not been addressed in the literature. In this paper, I propose an axiomatic approach, derived naturally from the revealed preference theory, that endogenously produces these reference preferences.

In order to explore my approach it is important to note that even though these behavioral anomalies are statistically significant, WARP still explains a sizable majority of the observations. ${ }^{9}$ As a result, and in order to keep the predictive power offered in the revealed preference theory to the extent possible, I focus on producing an axiomatic approach to decision making whose distance from the classical theory is proportionate to the extent that

\footnotetext{
${ }^{7}$ Our approach to status-quo bias is similar to that of phantom alternatives. A phantom alternative is an alternative which, for example because of being out of stock, is in the consideration set of the DM but can not be chosen. The significance of phantom alternatives are documented in the experimental works. See for example Doyl et al. (1999) for the case of phantom decoys.

${ }^{8}$ See, for example, Hardie, Johnson, and Fader (1993), Köszegi and Rabin (2006), Köszegi and Rabin (2007), and Carbajal and Ely (2016), Ok, Ortovela, and Riella (2011).

${ }^{9}$ In Huber et al. (1982) the distortion of WARP due to attraction effect ranges from $2 \%$ to $16 \%$ with the average of $9.2 \%$ across different treatments. Doyl et al. (1999)'s result on attraction effect also shows a range between $17 \%$ and $23 \%$. For phantom decoys the reported effect in this paper is $16 \%$. In Knetsch (1989) inducing initial endowments is reported to cause an average distortion of $39 \%$, relative to no initial endowment. The magnitude of status quo bias in Samuelson and Zeckhauser (1988) is $17 \%$.
} 
WARP is violated in the data. To do this, I propose the fundamental innovation of this paper which is embodied in a WARP decomposition theorem. I show that, indeed, WARP is decomposed into pairwise transitivity, top-down, and bottom-up rationales. In order to find the metric with which I need to deviate from WARP, next I search for those rationales that preclude the emergence of the referential effect. This is easy using earlier examples. Consider the example of attraction effect ${ }^{10}$ in which a DM has a pairwise transitive choice which is of the form $x \sim^{p} y \succ^{p} r$. Employing the reference preference $\succsim^{r}$, she breaks the indifference in favor of $x\left(x \succ^{r} y\right)$ which is the choice from the menu $x y r$. Therefore, the choice satisfies the top-down rationale; that is the choice in the the menu $x y r, x$, is at least as good as any other element in pairwise comparison. However, the choice does not satisfy the bottom-up rationale; though $y$ is at least as good as any other element in all possible pairs, $y$ does not survive in the larger menu. This, naturally, leads to the notion of referential decision maker, who only violates the bottom-up rationale at the referential level (that is the tripletons where a reference is relevant in the relative ranking of a pair), and is embodied in classical decision maker who fully admits WARP. This is illustrated in Figure 4. As shown in this figure, referential revealed preference theory extends the classical revealed preferences theory to the case where the choice behavior of the DM is explicitly pinned down by her action over doubletons and tripletons. In particular, and from the observations over doubletons and tripletons, the DM reveals a pairwise preference, $\succsim^{p}$, (which matches the same notion in the classical revealed preference theory), references, and a references relations. References are revealed wherever indifference break-down takes place. A reference relation, $\succsim^{r}$, is also revealed by observing the relative ranking $r$ induces on the referential level. ${ }^{11} \mathrm{I}$ show that each reference relation is complete and acyclic (abbreviated by RA in Figure 4), and therefore, attains a maximum. The final result of the paper is a full characterization of the choice behavior of the referential DM. This result states that the choice, in any arbitrary set, consists of those element(s) that posses the maximum number of reference(s) declaring them as the most preferred alternative.

\section{$1.1 \quad$ Related Literature}

WARP anomalies are not restricted to the mentioned effects and the body of the theoretical literature that addresses them is vast. These works include, but are not limited to, Shafir,

${ }^{10}$ The argument is similar for the status quo example.

${ }^{11}$ Therefore the referential revealed preference theory offers a rational treatment to the WARP anomalies. This is, from the methodological point of view, different from the behavioral approaches in addressing these effects. My approach also differs from the mentioned theories in terms of prediction. For a discussion on this see Section 5.3 . 

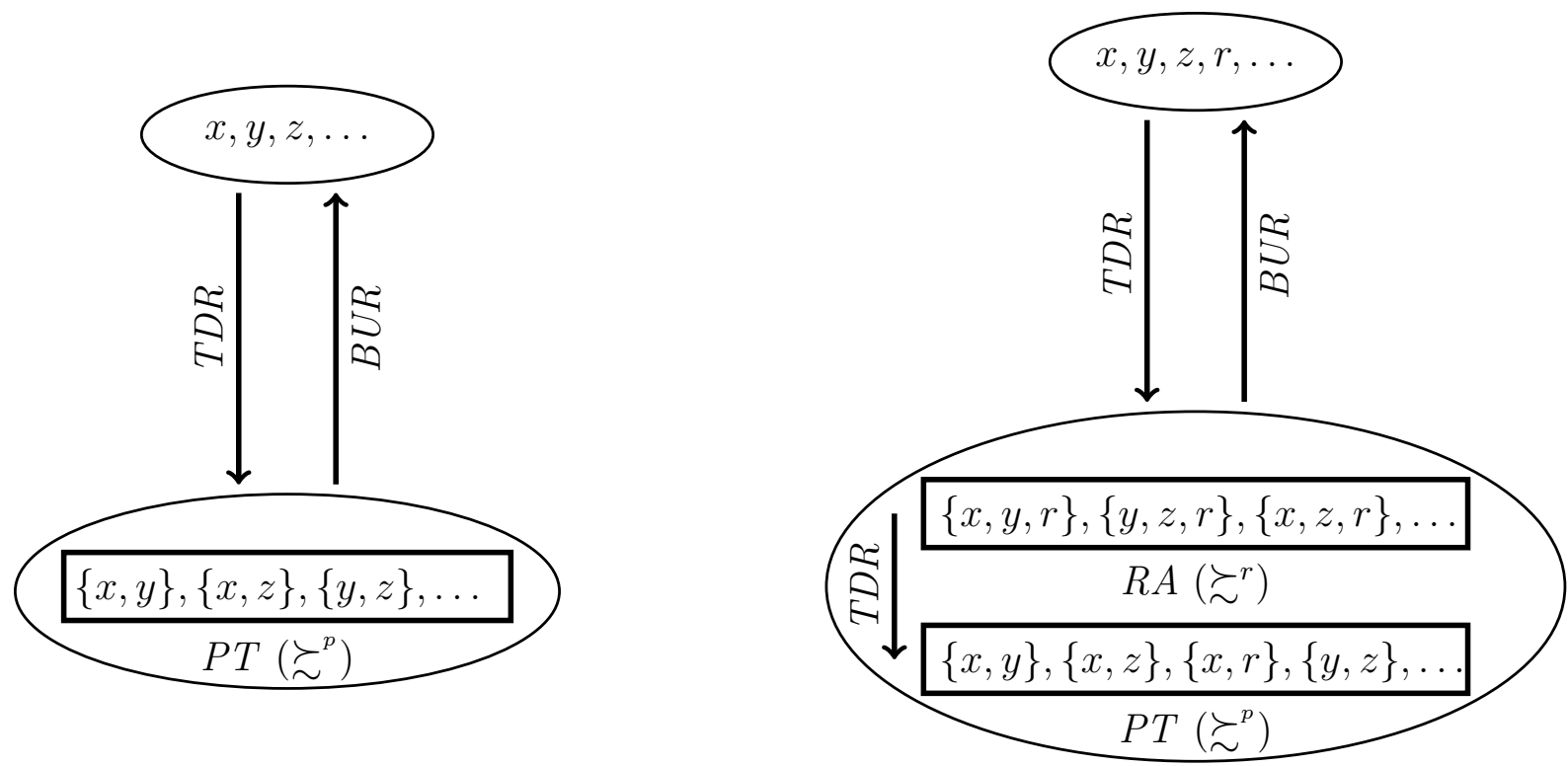

Figure 4: Classical DM (Left) and Referential DM (Right)

Simonson, and Tversky (1993), Tversky and Simonson (1993), Masatlioglu and Ok (2005), Kalai, Rubinstein, and Spiegler (2002), Rubinstein and Salant (2006), Manzini and Mariotti (2007), Salant and Rubinstein (2008), Kamenica (2008), Lombardi (2009), de Clippel and Eliaz (2012), Masatlioglu, Nakajima, and Ozbay (2012), Bordalo, Gennaioli, and Shleifer (2013), Cherepanov, Feddersen, and Sandroni (2013), Apesteguia and Ballester (2013), Ok et al. (2015), Lleras, Masatlioglu, Nakajima, and Ozbay (2015), and Gerasimou (2016). My approach is distinguished from the existing literature via decomposition theorem that enables a minimal deviation from WARP. Therefore, just like the classical theory, choice is completely characterized in the referential revealed preference theory. In what follows I explore the relation of my results to those in the literature that carry the closest relations.

Manzini and Mariotti (2007) (and in a similar manner Apesteguia and Ballester (2013)) takes an interesting approach where a DM's choice behavior is rationalized using a sequential procedure with two preferences. These authors concentrate on cyclical choice, however, and, therefore, only consider choice functions. Nonetheless, the application of their model to choice correspondence can also explain the anomalies of the nature of menu effect. In this regard, I show that, interestingly, the methodology of sequential thinking is endogenously derived from my approach. The two preferences $P_{1}$ and $P_{2}$ in these author's work can essentially be considered as $\succsim^{p}$ and $\succsim^{r}$ for any reference $r$.

The notion of multiple rationales in Kalai et al. (2002) is closely connected to the reference-dependent model in Tversky and Kahneman (1991). These rationales can be thought of as reference preference revealed in my model. However, this work is an effort 
to endogenize these reference preferences as opposed to their focus which is to find a minimal number of exogenously given rationales with which the choice behavior is rationalized. Similarly in Cherepanov et al. (2013) the concept of rationales are exogenously given. However, referential revealed preference theory produces results which are consistent with theirs. To see this once again, we can interpret the rationales as reference preferences in my model. Following their motivating example, assume that a decision maker who is choosing from the alternatives $\{x, y, z\}$ has the two following rationales (reference preferences): $x \succ^{r_{1}} y \succ^{r_{1}} z$ and $z \succ^{r_{2}} y \succ^{r_{2}} x$. As my model predicts $y$ can not be the choice in the menu $x y z$ as it is not a maximum element under any of the reference preferences. This matches the prediction in Cherepanov et al. (2013) as choosing $y$ is not rationalizable with respect to any of the rationales regardless of the structure of DM's innate preference. ${ }^{12}$

Masatlioglu and Ok (2005) proposes an axiomatic approach to the choice under status quo bias. Our model extends their model to the case where the DM confronts multiple status quo biases. This, for instance, results in nullifying the bias when DM has two opposing status quo points. To clarify, assume that the decision maker in the status quo example (Figure 3 is making a choice between $x$, and $y$ in time 2 , when she has already been endowed with both $x, y$ in time 1 . It would be natural to assume that the status quo bias (or the endowment effect) is nullified in such a scenario. Such effect is implied in my model, but rejected via SQB (or SQB*) axiom in Masatlioglu and Ok (2005). This difference between the two theories is that the status quo bias in Masatlioglu and Ok (2005) is modeled as a behavioral phenomenon, as opposed to the rational treatment offered in this paper. ${ }^{13}$

A different approach is to introduce the behavioral notion of constrained consideration. Masatlioglu et al. (2012) (and similarly Lleras et al. (2015)) is a predominant paper in this category. ${ }^{14}$ The main idea of the approach introduced in these papers is to add behavioral postulates to the analysis of choice. This is translated to an attention filter in Masatlioglu et al. (2012) (or the more general notion of consideration mapping in Lleras et al. (2015)) that limits the consideration set of the DM. The approach taken in Ok et al. (2015) is akin to that of the aforementioned paper. In these authors' work the consideration constraint is imposed via a reference map and an attraction correspondence that operates on the reference mapping. These features can be incorporated in my design if one thinks of a reference as the source of inattention and the set of maximum elements induced by it as the consideration

\footnotetext{
${ }^{12}$ The concept of innate preference in Cherepanov et al. (2013) is a fixed preference which is only used if it matches a rationale, however, I distance myself from such concept and allow for breaking down indifference via references. This difference has not relevance in terms of theoretical ramifications of these two theories.

${ }^{13}$ The mentioned manner with which the behavioral anomalies can by nullified via adding a extra opposing reference has also been experimentally documented. For example see Teppan and Felfering (2012).

${ }^{14}$ In addition to attraction effect, Masatlioglu et al. (2012) is also concerned with cyclical choice and choosing pairwisely unchosen. Lleras et al. (2015) is concentrated on choice overload phenomenon.
} 
set under that reference point. The idea of endogenous formation of references through weakening WARP is originated in Ok et al. (2015). Following their motivation and extending their results, the nature of referential effect, the relative place of the reference preferences to the classical revealed preference, reference preferences, and characterization of choice are also endogenously derived in this work. Some predictions of the theory developed here, however, are different from these works. Most importantly, it is natural in my model that a DM who admits a pairwise preference relation (complete and transitive) is not "unaware" of her most preferred alternatives; that is, she is never inattentive towards a dominant alternative in favor of a dominated one. ${ }^{15}$

The structure of this paper is as follows: Section 2 provides notations and basic definitions to be used in this paper. Section 3 builds the axiomatic foundations for the decomposition theorem, which is stated in Section 4. In Section 5, I introduce the notions of classical and referential DMs and explore some basic properties of the latter. The main results of the paper are presented in Section 6. Finally, Section 7 provides a conclusion.

\section{Preliminaries}

Let $X$ be a finite set. $X$ is the set of all "relevant" alternatives for the DM. Therefore, it contains not only the concrete options available to the DM, but also, for example, alternatives that she has chosen before, or phantom alternatives that are not available to choose but presented to her (e.g., items that are out of stock, or shows that are sold out). In terms of the nature of the elements, $X$ might be alternatives available for grocery shopping, different colleges to attend, various policies to be followed by the policy maker, etc. Let $2^{X}$ be the power set of $X$. Also let

$$
\mathfrak{X}^{k}:=\{A \subseteq X:|A|=k\}
$$

that is the set of all subsets of $X$ with cardinality equal to $k$, and

$$
\mathfrak{X}^{\geq k}:=\{A \subseteq X:|A| \geq k\}
$$

that is the set of all subsets of $X$ with cardinality of at least $k$. In order to simplify the domain of the discussion on choice I only consider the sets that have at least two elements, as the choice over the empty set and the singletons are trivially interpreted. Therefore let $\mathfrak{X}:=\mathfrak{X} \geq 2$. A choice correspondence on $X$ is a function $c: \mathfrak{X} \rightarrow 2^{X}$ such that for all $A \in \mathfrak{X}$ we have $\boldsymbol{c}(A) \subseteq A$. $\boldsymbol{c}$ is called a non-empty valued choice correspondence if $\boldsymbol{c}(A) \neq \varnothing$ for all

\footnotetext{
${ }^{15}$ For a detaled disucction of this see Section 5.3 .
} 
$A \subseteq X$. We make the common notational abuses:

$$
\boldsymbol{c}\{x, y, z\}:=\boldsymbol{c}(\{x, y, z\}) \text { and } \boldsymbol{c}\{x, y\}:=\boldsymbol{c}(\{x, y\}),
$$

for all $x, y, z \in X$.

Let $S \subseteq X$. Unless otherwise stated, whenever used throughout this paper let $S \in \mathfrak{X}^{\geq 3}$; that is let $S$ have at least three elements. Similar to $X$, for $S$ let

$$
\mathfrak{S}^{k}:=\{A \subseteq S:|A|=k\} \quad \text { and } \quad \mathfrak{S}^{\geq k}:=\{A \subseteq S:|A| \geq k\}
$$

For $x \in S$ let $S-x:=S \backslash\{x\}$; that is the set which is derived by removing $x$ from $S$.

A binary relation $R$ on $X$ is a subset of $X \times X$. Let $\mathcal{R}$ be the asymmetric relation derived from $R$; that is

$$
x \mathcal{R} y \Longleftrightarrow x R y \text { and } \neg(y R x) .
$$

A cycle of order $k$ in $R$ is a set $\left\{x_{1}, x_{2}, \ldots, x_{k}\right\}$ with $x_{i} \in X$ such that

$$
x_{1} \mathcal{R} x_{2} \mathcal{R} \ldots \mathcal{R} x_{k} \mathcal{R} x_{1} .
$$

$R$ is said to be acyclic if it does not posses any cycle of any order. A preference relation on $X$ is a binary relation which is transitive and complete. For a binary relation $R$ on $X$, and $S \subseteq X, x$ is called a maximum element of $R$ on $S$ if

$$
x R y: \forall y \in S .
$$

Let

$$
\underset{S}{\operatorname{argmax}} R:=\{x \in S: x \text { is a maximum for } R \text { on } S\} ;
$$

$x$ is called a maximal element of $R$ on $S$ if there does note exist $y \in A$ such that $y \mathcal{R} x$, where $\mathcal{R}$ is the asymmetric relation derived from $R$.

A cover for $S \subseteq X$ is a family of sets, $\left\{A_{i}\right\}_{i=1}^{n}$ such that $A_{i} \subseteq S$ for all $i$ and

$$
S=\bigcup_{i=1}^{n} A_{i}
$$

For a choice correspondence $\boldsymbol{c}$ define the relation $\succsim^{p}$ on $X$ by

$$
x \succsim^{p} y \Longleftrightarrow x \in \boldsymbol{c}\{x, y\} .
$$


Let $\succ^{p}$ and $\sim^{p}$ be asymmetric and symmetric parts of $\succsim^{p}$. Note that $\succsim^{p}$ matches the notion of revealed preference in the sense of Samuelson (1938). We call $\succsim^{p}$ the pairwise revealed preference throughout this paper. We next define the key notion of references.

Definition 1. (References) For a choice correspondence $\boldsymbol{c}$ and $S \subseteq X$ we say $r$ is a revealed reference ${ }^{16}$ in $S$ if there exits two distinct elements $x, y \in S$, both different from $r$ such that

$$
c\{x, y, r\} \subset c\{x, y\} .^{17} \text {. }
$$

Note that references are not chosen in sets where they operate; that is $r \notin \boldsymbol{c}\{x, y, r\}$ in the previous definition. Let

$$
\mathfrak{R}(S)=\{r \in S: r \text { is a reference in } S\}
$$

that is the set of references in $S$.

Assume that a decision maker rationalizes her choice in a menu by her relative choice in submenus. Recall from the intuitive introductory argument that, wherever operational, reference are not elements of choice themselves and only relevant by breaking the indifference. ${ }^{18}$ Therefore assume that submenus are derived by removing references from the original menu, one at a time, to the extent that referential effect is preserved; This creates a family of first-order diminished subsets that constitutes a cover for the original set. To see this in a formal setting, let

$$
R_{1}(S)=\{S-x: x \in \mathfrak{R}(S)\}, \text { and, } R_{2}(S)=\{S-x: x \in S \backslash \mathfrak{R}(S)\} .
$$

$R_{1}$ is the family of first-order diminished subsets of $S$ derived from removing references, one at a time, from $S . R_{2}$, in a similar fashion, is the family derived from removing non-reference

\footnotetext{
${ }^{16}$ The term is originally introduced in Ok et al. (2015). Recall from Figure 4 that, akin to the method in the revealed preference theory where the pairwise revealed preference characterized the choice, reference preferences provide a complete characterization of choice in my model. This provides a practical use of the word "revealed" as references (and also reference preferences) are, indeed, observable from the choice over doubletons and tripletons. However, for the sake of parsimony in writing, I drop the term "revealed" for the rest of this paper.

${ }^{17} \mathrm{I}$ am borrowing the definition of references from Ok et al. (2015). They introduce two different types of reference in their work. The first type captures the choice reflects breaking the indifference. For example, where $\boldsymbol{c}\{x, y\}=\{x, y\}, \boldsymbol{c}\{x, y, r\}=\{x\}$. The second type is where a DM, for example, has the following choice pattern: $\boldsymbol{c}\{x, y\}=\{y\}, \boldsymbol{c}\{x, y, r\}=\{x\}$. I interepret this as a DM who is indecisvie between the two alternatives $x, y$ when confronted with the menu $x y$, randomizing to choose $y$. Using this intrepretation the implied choice over the menu $x y$ is $\{x, y\}$ in the second definition. Therefore, the first definiton, which I use in this paper, captures both phenomena. Throughout this paper, and to keep the consistency in writing, I refer to such randomly resolved indecisiveness as indifference as well.

${ }^{18}$ This is naturally derived from my model. See Corollary 15 in Appendix.
} 
elements. We say $R_{1}(S)$ is referential if for all $A \in R_{1}(S)$ we have

$$
A \cap \mathfrak{R}(S) \neq \varnothing
$$

Next let

$$
\mathfrak{B}(S)= \begin{cases}R_{1}(S) & R_{1}(S) \text { is referential } \\ R_{2}(S) & R_{1}(S) \text { is not referential }\end{cases}
$$

To explore the nature of $\mathfrak{B}(S)$ and why it forms a cover for $S$ let us consider the following cases:

(i) If $\mathfrak{R}(S)=\varnothing$ then there is no reference in $S$ and therefore $A \cap \mathfrak{R}(S)=\varnothing$ for all $A \in$; that is $R_{1}(S)$ is not referential. Therefore, $\mathfrak{B}(S)=R_{2}(S)$. To show that $\mathfrak{B}(S)$ is a cover take $x \in S$. Since $|S| \geq 3$ there exists $y \in S$ different from $x$ and $S-y \in \mathfrak{B}(S)$. Obviously $x \in \boldsymbol{c}(S-y)$.

(ii) If $\mathfrak{R}(S)=\{r\}$ then removing the unique reference $r$ from $S$ results in first-order diminished subsets which lack references. Therefore, $R_{1}(S)$ is not referential and $\mathfrak{B}(S)=R_{2}(S)$ and all elements of $\mathfrak{B}(S)$ are derived by taking an element different than $r$ out of $S$; that is $r$ is in all elements of $\mathfrak{B}(S)$. To show that $\mathfrak{B}(S)$ is a cover take $x \in S$. If $x=r$ then $x \in A$ for all $A \in \mathfrak{B}(S)$ and therefore $\mathfrak{B}(S)$ is a cover for $S$. So assume $x \neq r$. Since $|S| \geq 3$ it follows that there exists a non-reference element $y \in S$ and $S-y \in \mathfrak{B}(S)$. Obviously $x \in S-y$.

(iii) If $|\mathfrak{R}(S)| \geq 2$ then removing a reference from the set leaves, at least, on other reference in $S$. Therefore $R-1(S)$ is referential and $\mathfrak{B}(S)=R_{1}(S)$. That is all elements of $\mathfrak{B}(S)$ are of the form $S-r$ for some reference $r$ in $S$. To show that $\mathfrak{B}(S)$ is a cover for $S$ take $x \in S$. If $x \in \mathfrak{R}(S)$ take a reference $r$ different from $x$ and note that $S-r \in \mathfrak{B}(S)$. Obviously $x \in S-r$. So assume $x$ is not a reference in $S$. Take a reference $r$ in $S$. It follows that $x \in S-r$.

As the argument above shows, the covers are built by removing the references from a menu, one at a time. If such procedure leads to blockage of the referential effect (that is when there is only one reference in the menu) the process is performed by removing non-referential elements. Obviously in the case of no references the cover contains all first-order diminished subsets. ${ }^{19}$

\footnotetext{
${ }^{19}$ The rationalizability motivation proposed here is particularly close to that of "divide and conquer" in Plott (1973). However, here, covers are not arbitrary and are specified via references.
} 
Definition 2. (Beating) Let $S \subseteq X$. For $x, y \in S$ we say $x$ beats $y$ in $S$ whenever $x \in \boldsymbol{c}(S)$ and $y \notin \boldsymbol{c}(S)$.

Conceptually, "beating" captures the idea of context-based choice. For example assume that a DM who is indifferent between $x, y$ choses $x$ under the reference $r_{1}$. Then $x$ beats $y$ in the context $\left\{x, y, r_{1}\right\}$. On the other hand, and under the reference $r_{2}$, the DM might choose $y$ in which case $y$ beats $x$ in the context of $\left\{x, y, r_{2}\right\}$. Therefore it is natural to assume that the DM is still not able to break the indifference between $x, y$ if presented to both $r_{1}$ and $r_{2}$. Building on beating, I next a develop a stronger notion which is a criteria for the $\mathrm{DM}$ to judge the relative importance of two alternatives. We refer to this latter notion as dominance.

Definition 3. (Dominance) Let $S \subseteq X$. We say $x$ dominates $y$ in $S$ and we write

$$
x \underset{S}{>} y
$$

if there exists $\bar{A} \in \mathfrak{B}(S)$ such that $x$ beats $y$ in $\bar{A}$, and there does not exist $A \in \mathfrak{B}(S)$ such that $y$ beats $x$ in $A$.

Note that dominance defines an asymmetric relation on $S .{ }^{20}$ Domination is a key notion in WARP decomposition which is developed in Section 4. It delivers a criteria for a DM to eliminate an alternative. To clarify, if $y$ is dominated by $x$, then it is never the case that DM choses $y$ over $x$ in all considered submenus. However, there is a submenu in her consideration where she choses $x$ over $y$. Therefore DM can not rationalize choosing $y$ using her relative choices on submenus as the criteria.

\section{Choice Axioms}

I start this section with a formal statement of WARP.

Axiom 0. (Weak Axiom of Revealed Preferences - WARP): We say a choice correspondence $\boldsymbol{c}$ satisfies WARP if for $S_{1}, S_{2} \subseteq X$ such that $x, y \in S_{1} \cap S_{2}$ we have

$$
x \in \boldsymbol{c}\left(S_{1}\right) \text { and } y \in \boldsymbol{c}\left(S_{2}\right) \text { implies } x \in \boldsymbol{c}\left(S_{2}\right) .
$$

Axiom 1. (Top-Down Rationality - TDR) We say $S$ satisfies TDR if

\footnotetext{
${ }^{20}$ To see this assume that $x y$. Then for all $A \in \mathfrak{B}(S)$ we have $y$ does not beat $x$ in $A$. This means $y{ }_{S} x$.
} 


$$
x \in \boldsymbol{c}(S) \Longrightarrow x \text { is a maximal element of } \longrightarrow \text {. }
$$

We say a choice correspondence $\boldsymbol{c}$ satisfies TDR on $S$ if all $S \in \mathfrak{S}^{\geq 3}$ satisfy TDR. If $S=X$, then we simply say $\boldsymbol{c}$ satisfies TDR.

Axiom 2. (Bottom-Up Rationality - BUR) We say $S$ satisfies BUR if

$x$ is a maximal element of $\longrightarrow x \in \boldsymbol{c}(S)$.

We say a choice correspondence $\boldsymbol{c}$ satisfies BUR on $S$ if all $S \in \mathfrak{S}^{\geq 3}$ satisfy BUR. If $S=X$, then we simply say $\boldsymbol{c}$ satisfies BUR.

Following the introductory discussion, TDR and BUR capture the notion of independence of irrelevant alternatives forced upon the DM via WARP. TDR requires the choice of the DM to be rationlizable with her choice on the submenus, where rationalizability is enforced via the notion of dominance. BUR, conversely, requires the rationlizable choice from the perspective of submenus, to survive in the larger menu.

The final ingredient of WARP decomposition is pairwise transitivity which is addressed in the following axiom.

Axiom 3. (Pairwise Transitivity - PT) We say a choice correspondence $\boldsymbol{c}$ satisfies PT on $S$ if for all $x, y, z \in S$

$$
x \succsim^{p} y \text { and } y \succsim^{p} z \text { implies } x \succsim^{p} z \text {. }
$$

If $S=X$, then we simply say $\boldsymbol{c}$ satisfies PT.

Note that PT implies transitivity of both $\succ^{p}$ and $\sim^{p}$. To conclude this section note that from the structure of my axioms it follows that if $\boldsymbol{c}$ satisfies TDR (resp. BUR, PT), then it satisfies TDR (resp. BUR, PT) on all $S \subseteq X$; that is these rationales are induced from larger menus to smaller ones.

\section{Decomposition Theorem}

However violated, WARP still rationalizes a good majority of observations in the experimental literature of behavioral anomalies. (See footnote9.) A natural manner to extend the revealed preference theory, therefore, would be to track those WARP-rationales which are the sole source of inability of the classical theory to explain the anomalies. This motivation distinguishes my axiomatic approach from those in the existing literature, as I extract axioms from WARP instead of introducing variations to it. This main innovation is presented in the following theorem. 
Theorem 1. (Decomposition Theorem) Let $\boldsymbol{c}$ be a choice correspondence. Then the following are equivalent:

(i) $\boldsymbol{c}$ is non-empty valued and satisfies WARP

(ii) $\boldsymbol{c}\{x, y\} \neq \varnothing$ for all $x, y \in X$ and $\boldsymbol{c}$ satisfies $T D R, B U R$, and PT.

The proof of Theorem 1 is presented in Section 8.1.1. Also, for independence of the axioms see Section 8.2 .

\section{Classical and Referential Decision Makers}

For the remainder of this paper assume $\boldsymbol{c}$ is a non-empty valued choice correspondence. Let $S \subseteq X$. For the sake of parsimony I use the following notation: $\mathcal{I}^{p}(S)=: \underset{S}{\operatorname{argmax}} \succsim^{p} \cdot \mathcal{I}^{p}(S)$ is, therefore, the set of best alternatives in $S$ from the perspective of the pairwise revealed preference. $S$ is called fully indecisive if $S \subseteq \mathcal{I}^{p}(S)$.

Definition 4. (Classical DM: CDM) A choice correspondence $\boldsymbol{c}$ is called a $C D M$ on $S$ if it satisfies TDR, BUR, and PT on $S$. If $S=X$, then we simply say $\boldsymbol{c}$ is a CDM.

Note that by decomposition theorem a CDM completely admits to WARP. Therefore the fundamental theorem of revealed preferences implies that the choice for CDM is completely pinned down by her transitive choice over doubletons. In the formal sense, if $\boldsymbol{c}$ is a CDM on $S$ then

$$
\boldsymbol{c}(S)=\mathcal{I}^{p}(S)
$$

that is a CDM's choice is not dependent on context. Obviously such contextlessness is violated in phenomena like status quo bias and attraction effect. In order to capture these contextual effects I next introduce the notion of referential DM.

\subsection{Referential Decision Maker}

As discussed in the introduction, the main source of the behavioral anomalies of concern in this paper is the BUR on tripletons where a reference operates. This next proposition formalizes a manner with which a minimal deviation from WARP (that is proportionate to the extent WARP is violated in data) can give rise to the referential effects of concern in this paper.

Proposition 1. Let $\boldsymbol{c}$ be a a choice correspondence which satisfies TDR and PT and $S \subseteq X$. Also let $\mathfrak{A}=\left\{A \in \mathfrak{S}^{3}\right.$ such that $A$ is not fully indecisive $\}$. Let $\boldsymbol{c}$ satisfy BUR on $\mathfrak{X} \geq 3 \backslash \mathfrak{A}$. Then 


$$
\text { c satisfies } B U R \text { on all } A \in \mathfrak{A} \Longleftrightarrow \mathfrak{R}(S)=\varnothing \text {. }
$$

The proofs of Proposition 1 is presented in Section 8.1.2. Note that $\boldsymbol{c}$ in this proposition has all the rationalities of a CDM except, possibly, BUR on referential tripletons. Proposition 1, therefore, simply states that the emergence of references in my model is equivalent to removing BUR on referential tripletons. Such relaxation, as a result, guarantees the proportionate deviation from WARP. Motivated by this proposition I next define referential DM. $^{21}$

\section{Definition 5. (Referential DM: RDM) Let}

$$
\mathfrak{A}=\left\{A \in \mathfrak{S}^{3} \text { such that } A \text { is not fully indecisive }\right\} \text {. }
$$

A choice correspondence $\boldsymbol{c}$ is called a $R D M$ on $S$ if

(i) $\boldsymbol{c}$ satisfies TDR and PT on $S$

(ii) $\boldsymbol{c}$ satisfies BUR for all $A \in \mathfrak{S}^{\geq 3} \backslash \mathfrak{A}$.

If $S=X$, then we simply say $\boldsymbol{c}$ is a RDM. ${ }^{22,23}$

\footnotetext{
${ }^{21}$ Relaxing BUR on fully indecisive tripletons, in fact, captures another behavioral anomaly referred to as compromise effect in the literature. This effect pertains to references that act on the element from the same indifference curve. However consistent with my framework, explaining this effect is beyond the scope of this work.

${ }^{22}$ Note that references are defined by breaking indifference. Therefore, if $\boldsymbol{c}$ is a choice function (that in the absence of indifference), then there are no references in $X$ and Proposition 1 implies that a RDM is a $\mathrm{CDM}$; that is the classical and referential revealed preference theories coincide in the absence of pariwise indifference.

${ }^{23}$ The notion of path independence in Plott $(1973)$ is related to CDM and RDM. We say a choice correspondence $\boldsymbol{c}$ satisifies path independence if for all $S \subseteq X$ and two finite covers of $S, v_{1}$ and $v_{2}$, we have

$$
c\left(\bigcup_{v \in v_{1}} c(v)\right)=c\left(\bigcup_{v \in v_{2}} c(v)\right) .
$$

To explore this relation first note that a CDM admits to WARP and therefore satisfies path independence. On the other hand, path independence does not imply WARP. To see this, consider the following example which is introduced in Plott (1973). Let $\boldsymbol{c}\{x, y, r\}=\{x, y\}, \boldsymbol{c}\{x, y\}=\{x, y\}, \boldsymbol{c}\{x, r\}=\{x, r\}$, and $\boldsymbol{c}\{y, r\}=\{y, r\}$. This choice structure satisfies path independence (see Plott (1973) for the proof), but it obviously violates WARP. There is no logical relation between RDM and a path independent choice structure however. To see this consider the following choice correspondence: $\boldsymbol{c}\{x, y, r\}=\{x\}, \boldsymbol{c}\{x, y\}=\{x, y\}, \boldsymbol{c}\{x, r\}=\{x\}$, and $\boldsymbol{c}\{y, r\}=\{y\}$. This is typical case of attraction effect and therefore consistent with RDM. However this choice correspondence does not satisfy path independence. To see this consider the two following covers: $v_{1}=\{\{x, y, r\}\}$ and $v_{2}=\{\{x, r\},\{y, r\}\}$. Then $\boldsymbol{c}\left(\bigcup_{v \in v_{1}} v\right)=\boldsymbol{c}\{x, y, r\}=\{x\}$ and $\boldsymbol{c}\left(\bigcup_{v \in v_{2}} v\right)=$ $\boldsymbol{c}(\boldsymbol{c}\{x, r\} \cup \boldsymbol{c}\{y, r\})=\boldsymbol{c}\{x, y\}=\{x, y\}$. To see that path independence does not imply RDM consider, again, the example from Plott (1973). Since $x \sim^{p} y \sim^{p} r$ we conclude that $\{x, y, r\}$ is fully indecisive. If $c$ was a RDM then we would have $\boldsymbol{c}\{x, y, r\}=\{x, y, r\}$ which is not possible.
} 
Note that it is obvious from the definitions that if $\boldsymbol{c}$ is a RDM (resp. CDM) then $\boldsymbol{c}$ is a RDM (resp. CDM) on all $S \subseteq X$. Next I explore some basic, yet critical, properties of a RDM. Recall from my motivational examples that references lie on indifference curves below the ones where they operate. One of the benefits of my approach via decomposition theorem is that such phenomenon is naturally derived from my setup. This next proposition, for which the proof is presented in Section 8.1.3, formalizes this observation.

Proposition 2. Let $\boldsymbol{c}$ be a RDM. Also let $r \in \mathfrak{R}(S)$ and $x, y \in S$ such that

$$
c\{x, y, r\} \subset \boldsymbol{c}\{x, y\}
$$

Then $x \sim^{p} y \succ^{p} r$.

Having defined both notions of CDM and RDM, an important question arises: How is the choice behavior of $R D M$ related to that of CDM? As discussed before CDM's choice is independent of context and summarized by $\boldsymbol{c}(S)=\mathcal{I}^{p}(S)$. For RDM, on the other hand, such contextlessness is violated. Nonetheless, RDM still satisfies TDR. This leads to a natural conjecture: RDM's choice must still be rationalizable by $\succsim^{p}$. This is in fact true and formalized in the next proposition.

Proposition 3. Let $\boldsymbol{c}$ be a $R D M$ and $S \subseteq X$. Then

$$
\boldsymbol{c}(S) \subseteq \mathcal{I}^{p}(S)
$$

The proofs of Proposition 3 is presented in Section 8.1.3. The intuition is quite simple. As long as DM is "capable" to reveal her rational preference in all pairs of alternatives, that is if $\succsim^{p}$ is a complete and transitive relation, then she is completely "aware" of all available options and the manner in which she ranks them. Therefore, there is no reason to ignore dominant alternatives in favor of dominated ones. ${ }^{24}$

\section{$5.2 \quad$ Reference Relations}

After observing the pairwise revealed preference $\left(\succsim^{p}\right)$ from doubletons, the next step is to observe where indifferences are broken as a result of adding a third alternative. This revelation of references is then followed by observation in regards to the manner references rank other elements, in particular those in a highest indifference curve, giving birth to revealed reference relations. ${ }^{25}$ This notion is introduced in the following definition.

\footnotetext{
${ }^{24}$ This intuitive result, indeed, is key to the distinction between predictions of my rational approach and the existing behavioral treatments in the literature. I elaborate on this point in Section 5.3.

${ }^{25} \mathrm{As}$ in the case of references, I drop the word "revealed" from the rest of this paper.
} 
Definition 6. (Reference Relation) Let $S \subseteq X$ and $r \in \mathfrak{R}(S)$. For two distinct element $x, y \in S$, both different from $r$, define

$$
x \succsim^{r} y \Longleftrightarrow x \in \boldsymbol{c}\{x, y, r\}
$$

Also let $\succ^{r}$ and $\sim^{r}$ be the asymmetric and symmetric parts of $\succsim^{r}$.

This next proposition explores the nature of the reference relations.

Proposition 4. Let $\boldsymbol{c}$ be a $R D M, S \subseteq X$, and $r \in \mathfrak{R}(S)$. Then

(i) $x \succsim^{r} y \Longrightarrow x \succsim^{p} y$.

(ii) $\succsim^{r}$ defines a complete binary relation on $\mathcal{I}^{p}(S)$.

The proof of Proposition 4 is presented in Section 8.1.4. Proposition 4 . i speaks to the evolution of the pairwise revealed preference by adapting to a reference point $r$. To see this let us look at an example that shows the reverse direction may not hold. Let $S=\{x, y, r\}$ such that $\boldsymbol{c}\{x, y\}=\{x, y\}, \boldsymbol{c}\{x, r\}=\{x\}, \boldsymbol{c}\{y, r\}=\{y\}$, and $\boldsymbol{c}\{x, y, r\}=\{x\}$. This is typical case of attraction effect (or statues quo bias). $\boldsymbol{c}$ is a RDM on $S$, and $y \succsim^{p} x$, but $x \succ^{r} y$. Proposition 44ii, on the other hand, guarantees one of the two essential characteristics of reference relations: completeness. ${ }^{26}$ As I show in Section 6 reference relations are also acyclic, completing their characterization. ${ }^{27}$

\subsection{Maximal References}

Definition 7. We say $r \in S$ is a maximal reference if there exists $x, y \in \mathcal{I}^{p}(S)$ such that

$$
\boldsymbol{c}\{x, y, r\} \subset \boldsymbol{c}\{x, y .\}
$$

We also use the following notation:

$$
\mathfrak{R}_{M}(S):=\{r \in S \text { such that } r \text { is a maximal reference in } S\} \text {. }
$$

\footnotetext{
${ }^{26}$ As discussed before for any arbitrary sets $S$, a RDM's behavior satisfies $\boldsymbol{c}(S) \subseteq \mathcal{I}^{p}(S)$ and, therefore, $\mathcal{I}^{p}(S)$ is the domain of relevance for $\succsim^{r}$. This intuition is in fact true; that is $\underset{S}{\operatorname{argmax}} \succsim^{r}=\underset{\mathcal{I}^{p}(S)}{\operatorname{argmax}} \succsim^{r}$. For the proof of this latter statement see footnote 38 in the Appendix.

${ }^{27} \succsim^{r}$ are assumed to be transitive in Tversky and Kahneman (1991). However, for the purpose of maximization the weaker notion of acyclicity of references is sufficient.
} 
Definition 8. For a maximal reference $r$ and $x \in S$ we say $r$ refers $x$ in $S$ (or equivalently, $r$ is a $x$-maximal reference) if

$$
x \in \underset{\mathcal{I}^{p}(S)}{\operatorname{argmax}} \succsim^{r}
$$

We also use the the notation:

$$
\mathfrak{R}_{M}^{x}(S):=\left\{r \in \mathfrak{R}_{M}(S): r \text { is a } x \text {-maximal reference }\right\}
$$

We call $\mathfrak{R}_{M}^{x}(S)$ the maximal reference set of $x$ in $S$.

Note that

$$
\mathfrak{R}(S) \supseteq \mathfrak{R}_{M}(S)=\bigcup_{x \in \mathcal{I}^{p}(S)} \mathfrak{R}_{M}^{x}(S) .
$$

The difference between the maximal and non-maximal references is illustrated in Figure 5. In this figure, brackets represent indifference classes (indifference curves) and the most preferred class, $\mathcal{I}^{p}(S)$, is the one of the left containing $x_{1}, x_{2}$, and $x_{3}$. Recall from the motivational examples (and also from Proposition 2 ) that references are from lower indifference curves and therefore all referential effects (presented by both dashed and solid arrows) are from a class on the right to one on the left. $x_{7}$ is a reference that breaks down the indifference between $x_{4}$ and $x_{5}$ in favor of $x_{4}$, however, it does not affect the indifference between the elements of the most preferred class and therefore not a maximal reference (these are shown by dashed arrows). $x_{6}$, and $x_{4}$, on the other hand, are maximal references since they affect the indifference in $\mathcal{I}^{p}(S)$ (these are shown by solid arrows). In particular, $\left\{x_{2}\right\}=\underset{\mathcal{I}^{p}(S)}{\operatorname{argmax}} \succsim^{x_{6}}$,

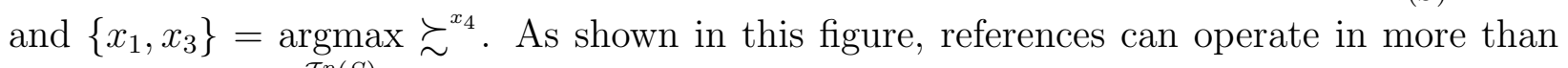
one class. For example, $x_{6}$ breaks the indifference in both first and second class. Also note that even though $x_{5}$ is dominated from the preceptive of pairwise comparison, it is not a reference since there is no observation of indifference break-down for this element. Finally, and obviously from my setup, elements of $\mathcal{I}^{p}(S)$ do not induce referential effects. Note that the notion of referring is only defined for maximal references. In Figure $5, x_{6}$ refers to $x_{2}$ and $x_{4}$ refers to $x_{1}$, and $x_{3}$.

A natural question arises here: why is the notion of referring only defined for maximal references? This is purposeful, and indeed, speaks to the key difference that distinguishes the rational treatment in referential revealed preference theory from the behavioral approaches taken in the existing literature. ${ }^{28}$ As I show in Section 6(Theorem 3) the maximal references

\footnotetext{
${ }^{28}$ See, for example, Masatlioglu et al. (2012), Ok et al. (2015), and Lleras et al. (2015). Masatlioglu et al. (2012), Lleras et al. (2015) only consider choice functions. Here I consider the implications of their approach on choice correspondence.
} 


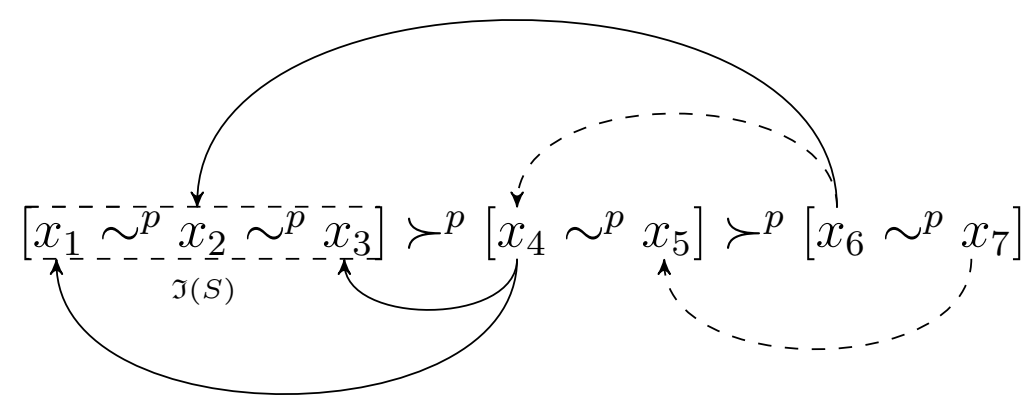

Non-Maximal Reference(s): $x_{7}$

Maximal References: $x_{4}, x_{6}$

Figure 5: Maximal and Non-Maximal References

are the sole determinants of the RDM's behavior that goes beyond WARP. Indeed, recall from Proposition 3 that RDM's behavior satisfies $\boldsymbol{c}(S) \subseteq \mathcal{I}^{p}(S)$. As a result only those references that effect the relative ranking of elements of $\mathcal{I}^{p}(S)$ play a role in the characterization of choice.

Let me consider an example. Assume that a DM is choosing from a menu in a restaurant. The menu consists of five options: i. beef ribs with a side of lentil soup, ii. pork ribs with side that does not contain lentil, nor beans, iii. a vegetarian dish (veg1) containing both lentil and beans, iv. a vegetarian dish (veg2) that does not contain lentil, nor beans, and v. vegan dish that contains beans. Assume that price is of no concern for the DM. DM is an absolute meat lover who, even though does not mind vegetarian dishes, will not choose them over meat options. Assume the following pairwise preference:

$$
\text { [beef ribs } \sim^{p} \text { pork ribs] } \succ^{p}\left[\operatorname{veg} 1 \sim^{p} \text { veg 2] } \succ^{p}\right. \text { [vegan]. }
$$

DM associates the variety of lentil and beans in the menu as a sign of chef's specialty and therefore veg1 and vegan dish acts as references. Now consider the two following scenarios:

Assume, in the first scenario, that the restaurant is out of ribs (both pork and beef). Then veg1 and veg2 are the most favored alternatives from the perspective of pairwise comparison. In this scenario, offering beans, vegan dish acts as a reference that effect the most favored class and therefore a maximal reference under which DM chooses veg1.

In the second scenario, all options in the menu are available. In this case, offering lentil, veg1 also acts as a reference and the following referential effects are observed:

Veg1 is a maximal reference (solid arrow) that favors beef ribs over pork ribs. However vegan dish is not a maximal reference anymore (dashed arrow) as it fails to effect the indifference between the most preferred options. The choice of veg1 in this scenario is consistent 


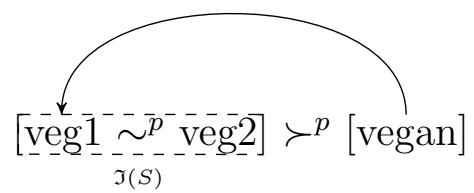

Scenario 1

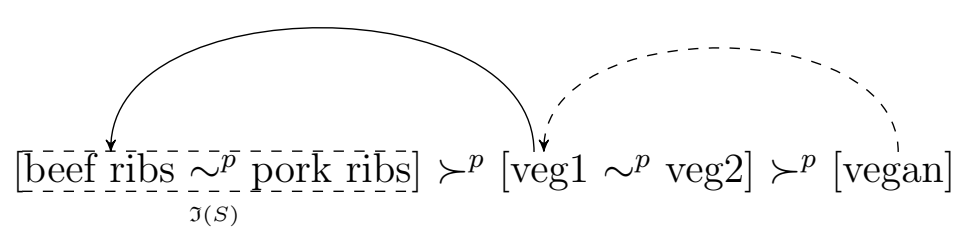

Scenario 2

if an arbitrary notion of "inattention" (or attraction) is employed. In words, a DM's might use the vegan dish as a reference and become inattentive towards the dominant options of meat, and therefore choose the dominated alternative veg1. However, such prediction is not consistent with the referential revealed preference theory; that is WARP deviations are only caused by maximal references and, therefore, the consistent choice in this scenario is beef ribs. In words, a RDM who admits to a complete and transitive ${ }^{29}$ preference relation on the entire set of alternative is not irrational in her inattention. ${ }^{30}$

\section{Main Results}

Now I can proceed to the main results of the paper. Recall form Proposition 4 ii that $\succsim^{r}$ is a complete relation on $\mathcal{I}^{p}(S)$. The first result completes the characterization of reference relations asserting that they are also acyclical. ${ }^{31}$

Theorem 2. (Acyclicity of Reference Relations) Let $\boldsymbol{c}$ be a $R D M$ and $r \in \mathfrak{R}(S)$. $\succsim^{r}$ defines an acyclic relation on $\mathcal{I}^{p}(S)$.

\footnotetext{
${ }^{29}$ This is also an assumption in the mentioned papers.

${ }^{30}$ To explore this distinction in more detail, let me employ the jargon of inattention and say veg1 attracts attention to beef ribs, and vegan dish attracts attention to veg1. Then DM chooses to be rational in her inattention by only using those references that affect her most preferred alternatives. The aforementioned interpretation is, in a sense, in line with the talking point of the rational inattention models introduced in Sims (2003). These models, however, are indistinguishable from the processing capacity constrain (Shannon entropy) under which the choice is made. This constraint is lacking in the current work, and therefore, to avoid confusion, I keep the jargon of this work akin to that of classical revealed preference theory.

${ }^{31}$ Reference acyclicity (RA) also appears in Ok et al. (2015). Two things are important to note here. The notion in these authors' paper refers to the manner references operates on each other and, therefore, different from the acyclic "revealed binary relation" induced here. Indeed, the two notions are not logically nested. Second, RA is an axiom in Ok et al. (2015) and a result in this paper.
} 
The proof of Theorem 2 is presented in Section 8.1.6. The final theorem in this paper is a characterization of choice on an arbitrary set with arbitrary number of references.

Theorem 3. Let $\boldsymbol{c}$ be a $R D M$ and $S \subseteq X$. Then $x \in \boldsymbol{c}(S)$ if and only if $x \in \mathcal{I}^{p}(S)$ and for all $t \in \mathcal{I}^{p}(S)$, the number of $x$-maximal references in $S$ is greater than or equal to the number of $t$-maximal references in $S$.

Proof. We need to show that

$$
x \in \boldsymbol{c}(S) \Longleftrightarrow x \in \mathcal{I}^{p}(S), \text { and }\left|\Re_{M}^{x}(S)\right| \geq\left|\Re_{M}^{t}(S)\right| \text { for all } t \in \mathcal{I}^{p}(S) \text {. }
$$

I start with following lemmas the proof of which, in order to keep the flow of the argument, are presented in Section 8.1.7.

Lemma 9. Let $\boldsymbol{c}$ be a $R D M$ and $S \subseteq X$ that possesses at least two references. There exists $\bar{r} \in \mathfrak{R}(S)$ such that $x \in \boldsymbol{c}(S-\bar{r})$.

Lemma 10. Let $\boldsymbol{c}$ be a $R D M$ and $S \subseteq X$ that possesses at least two maximal references. For any reference $r$ in $S$ assume

$$
t^{*} \in \boldsymbol{c}(S-r) \Longleftrightarrow\left|\mathfrak{R}_{M}^{t^{*}}(S-r)\right| \geq\left|\mathfrak{R}_{M}^{t}(S-r)\right|, \text { for all } t \in \mathcal{I}^{p}(S-r .)
$$

Also assume that $x \in \boldsymbol{c}(S)$ and $x, y \in \boldsymbol{c}(S-\bar{r})$ for a reference $\bar{r}$ in $S$. If $\bar{r}$ refers to $y$ then it refers to $x$.

We now, start the proof of the theorem by considering two cases.

$$
\text { Case 1: } \mathfrak{R}_{M}(S)=\varnothing \text {. }
$$

Note that in this case all elements in $\mathcal{I}^{p}(S)$ posses zero maximal references. Take $x \in \boldsymbol{c}(S)$. From Proposition 3 we have $x \in \mathcal{I}^{p}(S)$. Also, in $S$, the number of $x$-maximal references (zero) is greater than or equal to the number of $t$-maximal references (zero), for all $t \in \mathcal{I}^{p}(S)$ which completes $\Rightarrow$ direction of the proof. Next assume $x \in \mathcal{I}^{p}(S)$ (and note that it is true in case that, in $S$, the number of $x$-maximal references is greater than or equal to the number of $t$-maximal references, for all $\left.t \in \mathcal{I}^{p}(S)\right)$. It directly follows from Lemma 16 that $x \in \boldsymbol{c}(S)$. This completes the proof of $\Leftarrow$ and, hence, the proof of the statement for Case 1. 
Case 2: $\mathfrak{R}_{M}(S) \neq \varnothing$

Assume $S$ at least has one maximal reference. We prove the statement by induction on $\left|\Re_{M}(S)\right|$. Note that since the set of maximal references in $S$ is non-empty we conclude the set of references in $S$ is also non-empty.

Induction Base: Let $\mathfrak{R}_{M}(S)=\{r\}$.

$(\Rightarrow)$ : Let $x \in \boldsymbol{c}(S)$. Since $r$ is the unique maximal reference in $S$ Lemma 19 implies $x \in \underset{\mathcal{I}}{\operatorname{argmax}} \succsim^{r}$. First note $x \in \mathcal{I}^{p}(S)$. Also, $r$, as the single maximal reference in $S$, refers $\mathcal{I}^{p}(S)$ to $x$ and the result follows.

$(\Leftarrow)$ : Now assume $x \in \mathcal{I}^{p}(S)$ and

$$
\left|\mathfrak{R}_{M}^{x}(S)\right| \geq\left|\mathfrak{R}_{M}^{t}(S)\right| \text { for all } t \in \mathcal{I}^{p}(S)
$$

We must show $x \in \boldsymbol{c}(S)$. Note that since $r$ is a maximal reference in $S$ Corollary 20 implies that $\underset{\mathcal{I}^{p}(S)}{\operatorname{argmax}} \succsim^{r} \neq \varnothing$. So take $z \in \underset{\mathcal{I}^{p}(S)}{\operatorname{argmax}} \succsim^{r}$. This means $r$ refers to $z$. Since the number of $x$-maximal references in $S$ is greater or equal to the number of $z$-maximal references, and $S$ only possesses a single maximal reference, $r$, we conclude $r$ refers to $x$. Therefore $x \in \underset{\mathcal{I}^{p}(S)}{\operatorname{argmax}} \succsim^{r}$. Note that $\boldsymbol{c}$ satisfies the conditions in Lemma 19 and we conclude from $\Leftarrow$ of that theorem that $x \in \boldsymbol{c}(S)$.

Induction Hypothesis: Assume that the statement is true for $A \subseteq X$ with $\left|\Re_{M}(A)\right|=k$ and let $|\mathfrak{R}(S)|=k+1$. Note that we have $|\mathfrak{R}(S)| \geq\left|\mathfrak{R}_{M}(S)\right| \geq 2$ in our induction. Therefore we have $\mathfrak{B}(S)=R_{1}(S)$. That is all elements of $\mathfrak{B}(S)$ are of the form $S-r$ for some $r \in \mathfrak{R}(S)$.

$(\Rightarrow)$ : Let $x \in \boldsymbol{c}(S)$. Note that Lemma 14 implies $x \in \mathcal{I}^{p}(S)$. From Lemma 9 we conclude $x \in \boldsymbol{c}(S-\bar{r})$ for some $\bar{r} \in \mathfrak{R}(S)$.

Recall that we must show that, in $S$, the number of $x$-maximal references is greater than or equal to the number of $t$-maximal references, for all $t \in \mathcal{I}^{p}(S)$. Consider the set $S-\bar{r}$. Note that $\mathcal{I}^{p}(S-\bar{r})=\mathcal{I}^{p}(S)$. Take $y \in \mathcal{I}^{p}(S)=\mathcal{I}^{p}(S-\bar{r}) \subseteq S-\bar{r}$. We consider two cases here: 
Case 1: $y \notin \boldsymbol{c}(S-\bar{r})$.

Since $x \in \boldsymbol{c}(S-\bar{r})$ and $y \notin \boldsymbol{c}(S-\bar{r})$ then induction hypothesis implies that

$$
\left|\mathfrak{R}_{M}^{x}(S-\bar{r})\right|>\left|\mathfrak{R}_{M}^{y}(S-\bar{r})\right|
$$

and therefore adding $\bar{r}$ to the set $S-\bar{r}$ does not increase the number of $y$-maximal references over the number of $x$-maximal references; that is,

$$
\left|\Re_{M}^{x}(S)\right| \geq\left|\Re_{M}^{y}(S)\right|
$$

and the proof in this case is complete.

Case 2: $y \in \boldsymbol{c}(S-\bar{r})$.

Note that since $x, y \in \boldsymbol{c}(S-\bar{r})$ induction hypothesis implies

$$
\left|\mathfrak{R}_{M}^{x}(S-\bar{r})\right|=\left|\mathfrak{R}_{M}^{y}(S-\bar{r})\right|
$$

Note that all the conditions of Lemma 19 are met (considering induction hypothesis) and therefore we conclude if $\bar{r}$ refers to $y$ then it also refers to $x$. This means adding $\bar{r}$ to the set $S-\bar{r}$ does not increase the number of $y$-maximal references over the number of $x$-maximal references; that is,

$$
\left|\mathfrak{R}_{M}^{x}(S)\right| \geq\left|\mathfrak{R}_{M}^{y}(S)\right|
$$

This complete the proof in this case and, in turn, the proof of $\Rightarrow$ of the theorem.

$(\Leftarrow)$ : Take $x \in \mathcal{I}^{p}(S)$ and assume that

$$
\left|\mathfrak{R}_{M}^{x}(S) \geq\right| \mathfrak{R}_{M}^{t}(S) \mid, \text { for all } t \in \mathcal{I}^{p}(S)
$$

We must show $x \in c(S)$. First note in our induction there are at least two references in $S$ and therefore $|S| \geq 4^{32}$ and, therefore, $S$ satisfies BUR. s a result in order to show $x \in \boldsymbol{c}(S)$ we prove that there does not exist $y \in S$ such that $y{ }_{S} x$; that is we show $x$ is a maximal element of $\vec{S}_{S}$ on $S$. To do this first recall that all elements of $\mathfrak{B}(S)$ are of the form $S-r$ for some

${ }^{32}$ To see this note that since $r$ is a maximal reference in $S$ there exists two distinct elements, $t_{1}, t_{2}$ in $\mathcal{I}^{p}(S)$, both different from $r$. Next since $|\mathfrak{R}(S)| \geq 2$ there exists $s \neq r$ in $S$. Since $s$ is a reference in $S$ it follows from Proposition 6 iv that $s \notin \mathcal{I}^{p}(S)$. This in turn means $t_{1}, t_{2} \succ^{p} s$ and there for $t_{1}, t_{2}$ are different from $s$. Therefore we conclude $t_{1}, t_{2}, r, s$ are four distinct elements. This means $|S| \geq 4$. 
reference $r$ is $S$. Therefore assume that there exist $y \in S$ and $r^{*} \in \mathfrak{R}(S)$ such that $y$ beats $x$ in $S-r^{*}$. In particular $y \in \boldsymbol{c}\left(S-r^{*}\right)$ and therefore by Proposition $3 y \in \mathcal{I}^{p}\left(S-r^{*}\right)=\mathcal{I}^{p}(S)$. Note that since in our induction $\left|\mathfrak{R}_{M}(S)\right| \geq 2$ it follows that there exists a maximal reference in $S-r^{*}$. Therefore induction hypothesis applies to this set and we conclude

$$
\left|\mathfrak{R}_{M}^{y}\left(S-r^{*}\right)\right|>\left|\mathfrak{R}_{M}^{x}\left(S-r^{*}\right)\right| .
$$

First note that this along with (1) implies $\left|\Re_{M}^{x}(S)\right|=\left|\Re_{M}^{y}(S)\right|$. Second note that since dropping $r^{*}$ lowers the number of maximal references of $x$ relative to $y$ it has to be the case that $r^{*}$ refers to $x$ and $r^{*}$ does not refer to $y$. Since the number of maximal references that refer to $x$ in $S$ is equal to the number of maximal references that refer to $y$ there must exist a maximal reference $r^{* *}$ such that $r^{* *}$ refers to $y$, and $r^{* *}$ does not refer to $x$. To complete the proof of this direction consider the set $S-r^{* *}$. Since $x, y$ have the equal number of maximal references in $S$ dropping $r^{* *}$ from $S$ should lower the number of $y$-maximal references below the number of $x$-maximal references; that is

$$
\left|\mathfrak{R}_{M}^{x}\left(S-r^{* *}\right)\right|>\left|\mathfrak{R}_{M}^{y}\left(S-r^{* *}\right)\right|
$$

By induction assumption this means $x$ beats $y$ in $S-r^{* *}$. Therefore $x$ is not dominated by $y$ and BUR implies that $x \in \boldsymbol{c}(S)$. This completes this direction of the proof.

\subsection{Final Comments}

Some implication of the final theorem are important to note. First, if there are no maximal references in the set $S$ then the result formulated in Theorem 3 is simplified to

$$
\boldsymbol{c}(S)=\mathcal{I}^{p}(S)
$$

that is in the absence of maximal references the referential revealed preference theory coincides with the classical revealed preference theory. As we discussed in Section 5.3 this is a key distinction between the rational treatment in this paper and the behavioral ones in the existing literature.

Second, assume that $r$ is the unique maximal reference in the set $S$. Then from Theorem 3 the choice is characterized by those elements of $\mathcal{I}^{p}(S)$ to which $r$ refers. That is:

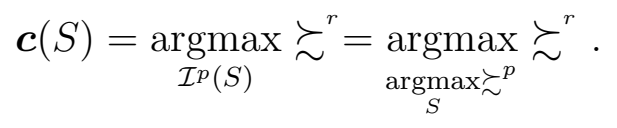


That is the choice in the set $S$ is rationalized by, first, applying $\succsim^{p}$ on $S$, and second, applying $\succsim^{r}$ on the resulting set. This is exactly the rational shortlist method (RSM) in Manzini and Mariotti (2007) applied to choice correspondence; that is, the methodology of sequential rationalizability arises, endogenously, in the referential revealed preference theory.

Third, it is implied from the result in Theorem 3 that opposing references nullify the referential effect. To clarify, assume that a DM who is indifferent between two items $x, y$ when confronted with the menu $x y$, prefers $x$ over $y$ when a reference $r_{1}$ is introduced into the menu, and $y$ over $x$ when an opposing reference $r_{2}$ is added. Therefore $r_{1}$, and $r_{2}$ are opposing maximal references that refer to $x$ and $y$, respectively, and Theorem 3 predicts that DM's choice on the menu $x y z r_{1} r_{2}$ should be $x y$; that is, she acts as if there were no references in the menu. This intuitive prediction is supported by the results in Teppan and Felfering (2012).

\section{Conclusion}

In this paper, I develop "revealed preference"-type theory (in the sense of Samuelson (1938)) that is consistent with the reference-dependent models (in the sense of Tversky and Kahneman (1991)). The main innovation of this paper is that I search for a minimal deviation from WARP (that is one which is proportionate to the extend WARP is violated in the data). This is done using the main innovation of this work which is a decomposition theorem of WARP. Therefore, instead of building a theory by introducing new axioms, I extract them from WARP. Next I track those WARP-rationals which are the sole reason of the inability of the classical theory in explaining behavioral anomalies such as attraction effect, statue quo bias. Removing these rationals from the classical DM, naturally, gives birth to the notion of the referential DM. I show that the rational treatment in this paper leads to more sensible predictions compared the existing behavioral ones in the literature. Interestingly, the methodology of sequential rationalizability in Manzini and Mariotti (2007) arises, endogenously, as an untapped potential in WARP coming to effect in the referential revealed

preference theory. I finally argue, employing decomposition theorem, my approach helps to keep the strong predictive power offered in the classical theory. That is, RDM's choice behavior is completely characterized by the observations on doubletons and tripletons (as compared to the classical revealed preference theory where such characterization is made with only observations on doubletons.) 


\section{Appendix}

\subsection{Proofs}

Throughout this section, and in order to avoid confusion, I preserve the symbol $\mathbf{\square}$ for Halmos Q.E.D. sign for the proofs of the main statements, and, wherever applied, $\square$ for subproofs. Let $\mathcal{F}$ be any of the operators $\mathfrak{R}, R_{1}, \mathfrak{B}$, or $\mathcal{I}^{p}$. I also make the following notational abuse throughout the arguments in this section.

$$
\mathcal{F}(\{x, y, z\}):=\mathcal{F}\{x, y, z\}
$$

For $S \subseteq X$, let

$$
S^{-}=\{A-x: x \in A\}
$$

Recall that $R_{1}(S)$ is the collection of of subsets of $S$ derived from taking references out of $S$, one at a time, and $R_{2}(S)$ is the collection of all subsets of $S$ derived from taking nonreference elements out. $S^{-}$, on the other hand, is the collection of all subsets of $S$ derived from removing any arbitrary element. Note that if $\mathfrak{R}(S)=\varnothing$ then $\mathfrak{B}(S)=R_{2}(S)=S^{-}$.

\subsubsection{Proof of Decomposition Theorem}

The following results are key to decomposition theorem.

Proposition 5. Let $\boldsymbol{c}$ be a choice correspondence that satisfies BUR and PT, and also assume $S \subseteq X$ such that $\boldsymbol{c}\{x, y\} \neq \varnothing$ for all $x, y \in S$. Then

(i) $\mathfrak{R}(S)=\varnothing$

(ii) $\mathfrak{B}(S)=S^{-}$.

Proof. (i) By contradiction assume there exists $r \in \mathfrak{R}(S)$. By definition there exists two distinct elements, $x, y \in S$, both different from $r$ such that

$$
\boldsymbol{c}\{x, y, r\} \subset \boldsymbol{c}\{x, y\}
$$

Obviously $r \notin \boldsymbol{c}\{x, y, r\}$. Since $\mathfrak{B}\{x, y, r\}$ is a cover for $\{x, y, r\}$ we conclude that $\{x, r\}$ or $\{y, r\}$ is in $\mathfrak{B}\{x, y, r\}$. Since $r \notin \boldsymbol{c}\{x, y, r\}$ BUR implies that there exists $t \in\{x, y\}$ such that $t \succ^{p} r$. Wlog, assume $t=x$.

Claim: $y \in c\{x, y\}$. 
Since $\boldsymbol{c}\{x, y\} \neq \varnothing$ assume $x \in \boldsymbol{c}\{x, y\}$. Note that since $x \in \boldsymbol{c}\{x, r\}$ BUR implies $x \in \boldsymbol{c}\{x, y, r\}$. From $\boldsymbol{c}\{x, y, r\} \subset \boldsymbol{c}\{x, y\}$ it follows that $\boldsymbol{c}\{x, y\}=\{x, y\}$ and therefore $y \in \boldsymbol{c}\{x, y\}$.

To finish the proof note that since $y \succsim^{p} x$ and $x \succ^{p} r$ PT implies that $y \succsim^{p} r$ which in turn by BUR implies $y \in \boldsymbol{c}\{x, y, r\}$. That is $\boldsymbol{c}\{x, y, r\}=\boldsymbol{c}\{x, y\}=\{x, y\}$ which is a contradiction.

(ii) Since $\mathfrak{R}(S)=\varnothing$ it follows from the definition of that $\mathfrak{B}(S)=R_{2}(S)=S^{-}$.

Lemma 11. (Quick Sort) Assume that a choice correspondence $\boldsymbol{c}$ satisfies PT, and let $S \subseteq X$ such that $\boldsymbol{c}\{x, y\} \neq \varnothing$ for all $x, y \in S$. Then there exists $x \in S$ such that $x \in \boldsymbol{c}\{x, y\}$ for all $y \in S$.

Proof. Let $|S|=k$. We need to prove that there exists $x \in S$ such that $x \succsim^{p} y$ for all $y \in S$. First note that since $\succsim^{p}$ is transitive then it follows that it is also acyclic. Now by contradiction assume that for all $x \in S$ there exists $y \in S$ such that $y \succ^{p} x$. Take $x_{1} \in S$. Then there must exist $x_{2} \in S$ such that $x_{2} \succ^{p} x_{1}$. Next there must exist $x_{3} \in S$ such that $x_{3} \succ^{p} x_{2} \succ^{p} x_{1}$. By repeating this procedure for $k$ times we conclude that

$$
x_{k+1} \succ^{p} x_{k} \succ^{p} \ldots \succ^{p} x_{1}
$$

where $x_{k+1} \in\left\{x_{1}, x_{2}, \ldots, x_{k}\right\}$. This produces a cycle in $\succsim^{p}$ which is a contradiction.

Lemma 12. Assume that a choice correspondence $\boldsymbol{c}$ satisfies TDR, BUR, and PT, and also assume $S \subseteq X$ such that $\boldsymbol{c}\{x, y\} \neq \varnothing$ for all $x, y \in S$. Then $\boldsymbol{c}(S) \neq \varnothing$, and

$$
x \in \boldsymbol{c}(S) \text { if and only if } x \in \boldsymbol{c}\{x, y\} \text { for all } y \in S^{33}
$$

Proof. Take $S \subseteq X$. First note that since $\boldsymbol{c}$ satisfies BUR, TDR, and PT Proposition 5 ii implies $\mathfrak{B}(S)=S^{-}$.

$(\Leftarrow)$ : We prove this by induction on $|S|$. For $k=2$ the statement is obvious. For $|k|=3$ let $S=\{x, y, z\}$. Then $x \in\{x, y\}$ and $x \in\{x, z\}$, by BUR, implies that $x \in \boldsymbol{c}(S)$. Now assume that for any set with cardinality $k$ the claim is true. For $S$ with caridnality $k+1$, let ${ }^{x} S^{-}=\left\{A \in S^{-}: x \in A\right\}$. Take $A \in{ }^{x} S^{-}$. Next assume $x \in \boldsymbol{c}\{x, y\}$ for all $y \in S$. This

\footnotetext{
${ }^{33}$ This Lemma is exatcly the axiom refered to as always chosen in Manzini and Mariotti (2007) which, in turn, captures the expansion axiom in these author's paper.
} 
means $x \in c\{x, y\}$ for all $y \in A$. Now by induction hypothesis we conclude $x \in \boldsymbol{c}(A)$. Since ${ }^{x} S^{-}$contains all elements of $S^{-}$that contain $x$, and $x$ is chosen in all such elements, we conclude $x$ is not dominated by any element in $S$ and from BUR it follows that $x \in \boldsymbol{c}(S)$.

$\boldsymbol{c}$ is non-empty valued: Let $S \subseteq X$.

Proof. First note since $\boldsymbol{c}$ satisfies PT then it follows from Quick Sort Lemma that there exists $x \in S$ such that $x \in \boldsymbol{c}\{x, y\}$ for all $y \in S$. Therefore by $\Leftarrow$ we conclude $x \in \boldsymbol{c}(S)$; that is $\boldsymbol{c}(S) \neq \varnothing$.

$(\Rightarrow)$ : We prove the statement by induction on $|S|$.

Induction Base: For $k=3$ let $S=\{x, y, z\}$ and assume $x \in \boldsymbol{c}(S)$. TDR implies that there does not exists $t \in S$ such that $t \rightarrow$. Since $\mathfrak{B}(S)=S^{-}$it follows that $x \in \boldsymbol{c}\{x, y\}$ and $x \in \boldsymbol{c}\{x, z\}$.

Induction Hypothesis: Let $|S|=k+1$ and assume the claim in true for all sets with cardinality $k$ (in particular for $A \in S^{-}$). Take $x \in \boldsymbol{c}(S)$ and an element $y \in S$ different from $x$. We must show $x \in \boldsymbol{c}\{x, y\}$. Let

$$
{ }^{x y} S^{-}=\left\{A \in S^{-}: x, y \in A\right\} .
$$

First note that since $|S| \geq 4$ in our induction it follows that ${ }^{x y} S^{-} \neq \varnothing .{ }^{34}$ If there exists $A \in{ }^{x y} S^{-}$such that $x \in \boldsymbol{c}(A)$ then by induction hypothesis we have $x \in \boldsymbol{c}\{x, y\}$ and the proof is complete. So suppose for all $A \in{ }^{x y} S^{-}$we have $x \notin \boldsymbol{c}(A)$.

Claim: $y \notin c(A)$ for all $A \in{ }^{x y} S^{-}$.

Proof. Take $A \in^{x y} S^{-}$. By contradiction assume $y \in \boldsymbol{c}(A)$. Note that since $x \notin \boldsymbol{c}(A)$ this means $y$ beats $x$ in $A$. Next since $x$ is never chosen in the elements of ${ }^{x y} S^{-}$, it follows that $x$ does not beat $y$ in all such elements. Therefore $y{ }_{S} x$ which is a contradiction since $x \in \boldsymbol{c}(S)$ and $\boldsymbol{c}$ satisfies TDR.

Now Take $A_{1} \in{ }^{x y} S^{-}$. By the previous claim $x, y \notin \boldsymbol{c}(A)$. Since $\left|A_{1}\right| \geq 3$, and

\footnotetext{
${ }^{34}$ To see this note that there exists an element $z \in S$ different from both $x, y$. It follows that $S-z \in S^{-}$. Obviously $x, y \in S-z$. This means $S-z \in{ }^{x y} S^{-}$.
} 
$\boldsymbol{c}\left(A_{1}\right) \neq \varnothing$ it follows that there exists an element $z \in A_{1}$, different from $x, y$, such that $z \in \boldsymbol{c}\left(A_{1}\right)$. Therefore we conclude $z$ beats $x$ and $y$ in $A_{1}$. The latter fact by induction hypothesis implies $z \in \boldsymbol{c}\{y, z\}$. From the former, since $x \in \boldsymbol{c}(S)$ and by TDR, we conclude that there exists $A_{2} \in S^{-}$such that $x$ beats $z$ in $A_{2}{ }^{35}$. Therefore by induction hypothesis we conclude $x \in \boldsymbol{c}\{x, z\}$. So we have $z \in \boldsymbol{c}\{y, z\}$ and $x \in \boldsymbol{c}\{x, z\}$ which in turn mean $z \succsim^{p} y$ and $x \succsim^{p} z$. Now PT implies $x \succsim^{p} y$ which in turn means $x \in \boldsymbol{c}\{x, y\}$.

Theorem 1. (Decomposition Theorem) Let $\boldsymbol{c}$ be a choice correspondence. Then the following are equivalent.

(i) $\boldsymbol{c}$ is non-empty valued and satisfies WARP

(ii) $\boldsymbol{c}\{x, y\} \neq \varnothing$ for all $x, y \in X$ and $\boldsymbol{c}$ satisfies $T D R, B U R$, and $P T$.

Proof. (ii) $\Rightarrow(i)$ : First note that from Lemma 12 we conclude $\boldsymbol{c}$ is non-empty valued. To prove that TDR, BUR, and PT imply WARP take $S_{1}, S_{2} \subseteq X$ and $x, y, \in S_{1} \cap S_{2}, x \in \boldsymbol{c}\left(S_{1}\right)$, and $y \in \boldsymbol{c}\left(S_{2}\right)$. We must show $x \in \boldsymbol{c}\left(S_{2}\right)$. Applying $\Rightarrow$ in Lemma 12 to the set $S_{1}$ we conclude that $x \in c\{x, y\}$ which in turn means $x \succsim^{p} y$. Applying $\Rightarrow$ in Lemma 12 to the set $S_{2}$ we conclude that $y \in \boldsymbol{c}\{y, t\}$ for all $t \in S_{2}$ which in turn means $y \succsim^{p} t$ for all $t \in S_{2}$. Now PT implies $x \succsim^{p} t$ for all $t \in S_{2}$. Therefore by $\Leftarrow$ in Lemma 12 we conclude $x \in \boldsymbol{c}\left(S_{2}\right)$.

$(i) \Rightarrow(i i)$ : Let $S \subseteq X$. First note that since $\boldsymbol{c}$ is non-empty valued obviously $\boldsymbol{c}\{x, y\} \neq \varnothing$ for all $x, y, \in S$.

Assume that $\boldsymbol{c}$ satisfies WARP. We first prove the following claim.

Claim: $\mathfrak{R}(S)=\varnothing$.

Proof. By contradiction assume $r$ is a reference in $S$. From definition it follows that there exists two distinct elements $x, y \in S$, both different from $r$, such that

$$
\boldsymbol{c}\{x, y, r\} \subset \boldsymbol{c}\{x, y\} .
$$

Obviously $r \notin \boldsymbol{c}\{x, y, r\}$. Since $\boldsymbol{c}\{x, y, r\} \neq \varnothing$ and $\boldsymbol{c}\{x, y, r\} \subset \boldsymbol{c}\{x, y\}$ it follows that $\boldsymbol{c}\{x, y\}=\{x, y\}$. Now WARP implies $\boldsymbol{c}\{x, y, r\}=\{x, y\}$. This is a contradiction.

Note that from the previous claim it follows that $\mathfrak{B}(S)=S^{-}$.

\footnotetext{
${ }^{35}$ Indeed, $A_{2}=S-y$.
} 


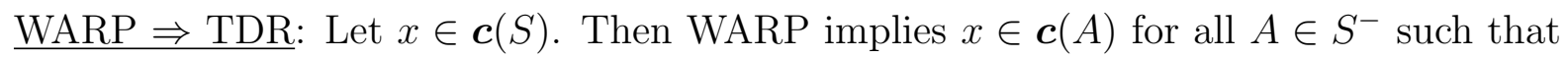
$x \in A$. Therefore, we conclude $x$ is not beaten by any elements of $S$ in any elements of $S^{-}$and, as a result, $x$ is not dominated by any elements of $S$. This means $x$ is a maximal element of

WARP $\Rightarrow$ BUR: Assume that $x$ is the maximal element of $\rightarrow$. We must show $x \in \boldsymbol{c}(S)$. Take $y \in \boldsymbol{c}(S)$ different from $x^{36}$. Let

$$
{ }^{x y} S^{-}=\left\{A \in S^{-}: x, y \in A\right\} .
$$

First note that ${ }^{x y} S^{-} \neq \varnothing .{ }^{37}$ Take $A_{1} \in{ }^{x y} S^{-}$. WARP (the equivalent statement) implies $y \in \boldsymbol{c}\left(A_{1}\right)$. If $x \in \boldsymbol{c}\left(A_{1}\right)$ then by WARP $x \in \boldsymbol{c}(S)$ and the proof is complete. So assume $x \notin \boldsymbol{c}\left(A_{1}\right)$. This means $y$ beats $x$ in $A_{1}$. Since $x$ is not dominated by $y$ it follows that there exists $A_{2} \in{ }^{x y} S^{-}$such that $x$ beats $y$ in $A_{2}$. In particular, $x \in \boldsymbol{c}\left(A_{2}\right)$. Now WARP implies $x \in \boldsymbol{c}(S)$.

WARP $\Rightarrow \mathrm{PT}$ : Take $x, y, z \in S$, and assume $x \succsim^{p} y$ and $y \succsim^{p} z$. These, respectively, mean $x \in \boldsymbol{c}\{x, y\}$ and $y \in \boldsymbol{c}\{y, z\}$. Consider the set $\{x, y, z\}$. Note the following cases: i. if $x \in \boldsymbol{c}\{x, y, z\}$ then WARP implies $x \in \boldsymbol{c}\{x, z\}$ and therefore $x \succsim^{p} z$. ii. If $y \in \boldsymbol{c}\{x, y, z\}$ WARP and $x \in \boldsymbol{c}\{x, y\}$ imply that $x \in \boldsymbol{c}\{x, y, z\}$ and by i. $x \succsim^{p} z$. iii. If $z \in \boldsymbol{c}\{x, y, z\}$ WARP and $y \in \boldsymbol{c}\{y, z\}$ imply that $y \in \boldsymbol{c}\{x, y, z\}$ which by ii. implies $x \succsim^{p} z$. Since $\boldsymbol{c}\{x, y, z\} \neq \varnothing$ we conclude $x \succsim^{p} z$ and the proof is complete.

\subsubsection{Proof of Proposition 1}

Proposition 1. Let $\boldsymbol{c}$ be a a choice correspondence which satisfies TDR and PT and $S \subseteq X$. Also let $\mathfrak{A}=\left\{A \in \mathfrak{S}^{3}\right.$ such that $A$ is not fully indecisive $\}$. Let $\boldsymbol{c}$ satisfy $B U R$ on $\mathfrak{X}^{\geq 3} \backslash \mathfrak{A}$. Then

$$
\text { c satisfies } B U R \text { on all } A \in \mathfrak{A} \Longleftrightarrow \mathfrak{R}(S)=\varnothing \text {. }
$$

Proof. $(\Rightarrow)$ : Assume that $\boldsymbol{c}$ satisfies BUR on $\mathfrak{A}$. Note that this means $\boldsymbol{c}$ satisfies BUR. Then from Proposition 5 i it follows that $\mathfrak{R}(S)=\varnothing$.

$(\Leftarrow)$ : We only need to show all non-fully indecisive tripletones satisfy BUR. To do this Let $\{x, y, z\} \in \mathfrak{A}$. To show that $\{x, y, z\}$ satisfies BUR, wlog, assume that no elements of $S$

\footnotetext{
${ }^{36}$ Note that if such $y$ does not exists, since $\boldsymbol{c}(S) \neq \varnothing$, we conclude $x \in \boldsymbol{c}(S)$ and the proof is complete.

${ }^{37}$ To see this note that since $|S| \geq 3$, there exists an element $z \in S$ different from $x, y$. Obviously $x, y \in S-z$, and therefore ${ }^{x y} S^{-} \neq \varnothing$.
} 
dominate $x$. We must show $x \in \boldsymbol{c}\{x, y, z\}$. First note that since there are no references in $S$ we have $\mathfrak{B}\{x, y, z\}=\{x, y, z\}^{-}$. Since $y$ and $z$ do not dominate $x$, it follows that $x \in \boldsymbol{c}\{x, y\}$ and $x \in \boldsymbol{c}\{x, z\}$, which respectively, mean $x \succsim^{p} y$ and $x \succsim^{p} z$. We show that $x \notin \boldsymbol{c}\{x, y, z\}$ reaches a contradiction. Assume $x \notin \boldsymbol{c}\{x, y, z\}$. Wlog, let $y \in \boldsymbol{c}\{x, y, z\}$. Then TDR implies that $y \in \boldsymbol{c}\{x, y\}$, which in turn means $y \succsim^{p} x$, and therefore $x \sim^{p} y$. Now PT implies $y \succsim^{p} z$; that is $y \in \boldsymbol{c}\{y, z\}$. If $z \notin \boldsymbol{c}\{x, y, z\}$ then we will have

$$
\boldsymbol{c}\{x, y, z\} \subset \boldsymbol{c}\{x, y\}
$$

and therefore $z$ will be a reference in $S$ which is not possible. So assume $z \in \boldsymbol{c}\{x, y, z\}$. Then TDR implies $z \in \boldsymbol{c}\{x, z\}$ which in turn means $z \succsim^{p} x$ and therefore $x \sim^{p} z$. From PT it follows that $x \sim^{p} y \sim^{p} z$ and therefore $\{x, y, z\}$ is fully indecisive. A contradiction.

\subsubsection{Proof of Propositions 2 and 3}

I start by the following basic observations about RDM.

Proposition 6. Let $\boldsymbol{c}$ be a RDM. The following is true:

(i) If $S$ is fully indecisive then $\boldsymbol{c}(S)=S$.

(ii) $|\mathfrak{R}\{x, y, z\}| \leq 1$

Proof. (i) We prove this by induction on $|S|$.

Induction Base: For $|S|=3$ let $S=\{x, y, z\}$. First note that since $S$ is fully indecisive it satisfies BUR. Take $t \in\{x, y, z\}$. First note that since $\mathfrak{B}\{x, y, z\}$ is a cover for $S$ and the fact that $x \sim^{p} y \sim^{p} z$ we conclude that $q t$ for all $q \in S$. Therefore, by BUR, we conclude $t \in \boldsymbol{c}(S)$. Therefore $\boldsymbol{c}\{x, y, z\}=\{x, y, z\}$.

Induction Hypothesis: Now assume that the statement is true if $|S|=k$. Let $|S|=k+1$. Take $x \in S$. Take $t \in S$. Note that for $A \in S^{-}$we have $A$ is fully indecisive and therefore by induction base $\boldsymbol{c}(A)=A$. Next note that $\mathfrak{B}(S) \subseteq S^{-}$. Therefore for all $A \in \mathfrak{B}(S)$ we have $\boldsymbol{c}(A)=A$. This implies there does not exists $q \in S$ such that $q \vec{S}_{t}$. BUR implies $t \in \boldsymbol{c}(S)$. This means $\boldsymbol{c}(S)=S$.

(ii) Consider the set $\{x, y, z\}$. By definition $t \in \mathfrak{R}\{x, y, z\}$ implies $t \notin \boldsymbol{c}\{x, y, z\}$. Since $\boldsymbol{c}\{x, y, z\} \neq \varnothing$ then we conclude $|\mathfrak{R}\{x, y, z\}|<3$. It suffice to show $|\Re\{x, y, z\}| \neq 2$. 
Assume, wlog, $\mathfrak{R}\{x, y, z\}=\{y, z\}$. First note that this means $\boldsymbol{c}\{x, y, z\}=\{x\}$. Next since $y, z$ are references we should have

$$
\{x\}=\boldsymbol{c}\{x, y, z\} \subset \boldsymbol{c}\{x, z\}
$$

and

$$
\{x\}=c\{x, y, z\} \subset c\{x, y\}
$$

which, respectively, imply

$$
c\{x, z\}=\{x, z\}
$$

and

$$
c\{x, y\}=\{x, y\}
$$

Next PT implies $x \sim^{p} y \sim^{p} z$ and therefore we conclude $\{x, y, z\}$ is fully indecisive and by part $(i)$ we conclude $\boldsymbol{c}\{x, y, z\}=\{x, y, z\}$ which is a contradiction. Therefore $|\Re\{x, y, z\}| \leq 1$.

Proposition 2, Let $\boldsymbol{c}$ be a RDM. Also let $r \in \mathfrak{R}(S)$ and $x, y \in S$ such that

$$
\boldsymbol{c}\{x, y, r\} \subset \boldsymbol{c}\{x, y\}
$$

Then $x \sim^{p} y \succ^{p} r$.

Proof. Take $r \in \mathfrak{R}(S)$ and $x, y \in S$ such that

$$
\boldsymbol{c}\{x, y, r\} \subset \boldsymbol{c}\{x, y\}
$$

First note that $r \notin \boldsymbol{c}\{x, y, r\}$, and, since $\boldsymbol{c}\{x, y, r\} \neq \varnothing$, it has to be the case that $\boldsymbol{c}\{x, y\}=$ $\{x, y\}$. Therefore $x \sim^{p} y$. Since $r$ is also a reference in $\{x, y, r\}$ from Proposition 6.ii we conclude $\mathfrak{R}\{x, y, r\}=\{r\}$. This implies

$$
\mathfrak{B}(S)=\{\{x, r\},\{y, r\}\}
$$

If, wlog, $r \in \boldsymbol{c}\{x, r\}$, then PT implies $x \sim^{p} y \sim^{p} r$ and therefore $\{x, y, z\}$ is fully indecisive and by Proposition 6. i we will have $\boldsymbol{c}\{x, y, z\}=\{x, y, z\}$ which is not possible. So it has to be the case that $\boldsymbol{c}\{x, r\}=\{x\}$ and $\boldsymbol{c}\{y, r\}=\{y\}$ which imply, respectively, $x \succ^{p} r$, and $y \succ^{p} r$.

This next statement is a direct result of the Proposition 2 and is used in the future results 
of the paper.

Corollary 13. Let $\boldsymbol{c}$ be a $R D M$ and $r \in \mathfrak{R}(S)$. Then $r \notin \mathcal{I}^{p}(S)$.

Proof. Take $r \in \mathfrak{R}(S)$. From the definition of reference we conclude that there exists two distinct elements $x, y, \in S$, both different from $r$ such that

$$
\boldsymbol{c}\{x, y, r\} \subset \boldsymbol{c}\{x, y\}
$$

Now Proposition 2 implies $x \sim^{p} y \succ^{p} r$. This means $r \notin \mathcal{I}^{p}(S)$.

Proposition 3. Let $\boldsymbol{c}$ be a $R D M$ and $S \subseteq X$. Then

$$
\boldsymbol{c}(S) \subseteq \mathcal{I}^{p}(S)
$$

Proof. We prove this by induction on $|S|$.

Induction Base: For $|S|=3$ let $S=\{x, y, z\}$. Let $x \in \boldsymbol{c}\{x, y, z\}$. We need to show $x \in \mathcal{I}^{p}\{x, y, z\}$. If $\mathfrak{R}(S)=\varnothing$ then $\mathfrak{B}(S)=\{x, y, z\}^{-}$and TDR implies that $x \in \boldsymbol{c}\{x, y\}$ and $x \in \boldsymbol{c}\{x, z\}$ which in turn implies $x \succsim^{p} y$ and $x \succsim^{p} z$. This means $x \in \mathcal{I}^{p}\{x, y, z\}$ and the proof is complete. So assume $\mathfrak{R}\{x, y, z\} \neq \varnothing$. Since $x \in \boldsymbol{c}\{x, y, z\}$ then the definition of reference implies $x \notin \mathfrak{R}\{x, y, z\}$. Also note that prp:properties.ii implies that there only exists one reference in $\{x, y, z\}$. Wlog, assume $\mathfrak{R}\{x, y, z\}=\{z\}$. Then Proposition 2 implies $x \sim^{p} y \succ^{p} z$ and therefore $x \in \mathcal{I}^{p}\{x, y, z\}$ and the proof is complete.

Induction Hypothesis: Now assume the statement is true if $|S|=k$. Let $|S|=k+1$. Assume $x \in \boldsymbol{c}(S)$ and take an element $y$ in $S$ different from $x$. We must show $x \succsim^{p} y$. Let

$$
{ }^{x y} \mathfrak{B}(S)=\{A \in \mathfrak{B}(S): x, y \in A\}
$$

Claim: ${ }^{x y} \mathfrak{B}(S) \neq \varnothing$.

Proof. By contradiction assume ${ }^{x y} \mathfrak{B}(S)=\varnothing$. Since $\mathfrak{B}(S)$ is a cover for $S$ there has to be a set in it that contain $x$, and a set that contains $y$. note that it follows from the contradiction that

$$
\mathfrak{B}(S)=\{S-x, S-y\} .
$$

Since $|S|>3$ and $\mathfrak{B}(S)$ only has two elements it has to be the case that $\mathfrak{B}(S)=R_{1}(S)$ which in turn means $\mathfrak{R}(S)=\{x, y\}$. 
Next note that $x \in \boldsymbol{c}(S-y)$. To see this, by contradiction assume $x \notin \boldsymbol{c}(S-y)$. Take $z \in \boldsymbol{c}(S-y)$. Since $x$ only appears in $S-y$ this implies that $z$ dominates $x$ in $S$ and therefore $x \notin \boldsymbol{c}(S)$ which is a contradiction. Therefore assume $x \in \boldsymbol{c}(S-y)$. Note that from induction assumption we conclude that $x \in \mathcal{I}^{p}(S-y)$. This means $x \succsim^{p} t$ for all $t$ in $S$ different from $y$.

Finally since $x \in \mathfrak{R}(S)$ it follows from the definition that there exist two distinct elements $t_{1}, t_{2} \in S$ both different from $x$, such that

$$
\boldsymbol{c}\left\{t_{1}, t_{2}, x\right\} \subset \boldsymbol{c}\left\{t_{1}, t_{2}\right\} .
$$

Now Proposition 2 implies $t_{1} \sim^{p} t_{2} \succ^{p} x$. Since $t_{1}, t_{2}$ are distinct elements, at least one of them is different from $y$. This contradicts our earlier observation that $x \succsim^{p} t$ for all $t$ different from $y$.

To finish the proof let ${ }^{x y} \mathfrak{B}(S) \neq \varnothing$. If $A \in{ }^{x y} \mathfrak{B}(S)$ exists such that $x \in \boldsymbol{c}(A)$ then by induction assumption we conclude $x \succsim^{p} y$ and the proof is complete. So assume for all $A \in{ }^{x y} \mathfrak{B}(S)$ we have $x \notin \boldsymbol{c}(A)$. First note that this implies $y \notin \boldsymbol{c}(A)$ for all $A \in{ }^{x y} \mathfrak{B}(S)$. To see this note that if $y$ is chosen on an element of ${ }^{x y} \mathfrak{B}(S)$, it would mean that $y$ beats $x$ in that set. And since $x$ is never chosen in the elements of ${ }^{x y} \mathfrak{B} x$ does not beat $y$ in any of those elements. This means $y \leq x$ which is not possible because of TDR and the fact that $x \in \boldsymbol{c}(S)$.

Therefore take $A_{1} \in{ }^{x y} \mathfrak{B}(S)$ and $z \in \boldsymbol{c}\left(A_{1}\right)$ different from $x, y$. Note that by induction assumption this implies $z \succsim^{p} y$. Next this implies that $z$ beats $x$ in $A_{1}$. Since $x \in \boldsymbol{c}(S)$ TDR implies that there exists $A_{2} \in \mathfrak{B}(S)$ such that $x$ beats $z$ in $A_{2}$. This from induction assumption on $A_{2}$ implies $x \succsim^{p} z$. Combining this latter fact with $z \succsim^{p} y$, from PT it follows that $x \succsim^{p} y$. This completes the proof of the proposition.

I finish this section by proving the following lemma and corollary that are used in the proof of the main results.

Lemma 14. Let $\boldsymbol{c}$ be a RDM. If $x \in \boldsymbol{c}\{x, y, z\}$ for all pair of distinct elements $y, z \in S$, both different from $x$, then $x \in \boldsymbol{c}(S)$.

Proof. We prove this by induction on $|S|$. For $|S|=3$ there is nothing to prove. Assume that the statement is true for all the sets with cardinality $k$. Let $|S|=k+1$ and take $x \in S$ be such that $x \in \boldsymbol{c}\{x, y, z\}$ for all two distinct elements $y, z \in S$, both different from $x$. Let

$$
{ }^{x} \mathfrak{B}(S)=\{A \in \mathfrak{B}(S): x \in A\} .
$$


Note that since $\mathfrak{B}(S)$ is a cover for $S$ we conclude ${ }^{x} \mathfrak{B}(S) \neq \varnothing$. Take $A \in{ }^{x} \mathfrak{B}(S)$. Note that since

$$
x \in \boldsymbol{c}\{x, y, z\},
$$

for two distinct elements $y, z \in S$ and, since $A \subseteq S$, we conclude that

$$
x \in \boldsymbol{c}\{x, y, z\},
$$

for two distinct elements $y, z \in A$, both different from $x$. Finally induction assumption implies that $x \in \boldsymbol{c}(A)$. Since $A$ was an arbitrary element of ${ }^{x} \mathfrak{B}(S)$ we conclude $x$ is chosen in all elements of ${ }^{x} \mathfrak{B}(S)$. This means $x$ is not dominated by any element of $S$. Therefore BUR implies that $x \in \boldsymbol{c}(S)$.

Corollary 15. Let $\boldsymbol{c}$ be a RDM. If $r \in \mathfrak{R}(S)$ then $r \notin \boldsymbol{c}(S)$.

Proof. Take $r \in \mathfrak{R}(S)$. Then Corollary 13 implies $r \notin \mathcal{I}^{p}(S)$. Next Proposition 3 implies $r \notin \boldsymbol{c}(S)$.

\subsubsection{Proof of Proposition 4}

Proposition 4. Let $\boldsymbol{c}$ be a $R D M$ and $r \in \mathfrak{R}(S)$. Then

(i) $x \succsim^{r} y \Longrightarrow x \succsim^{p} y$.

(ii) $\succsim^{r}$ defines a complete binary relation on $\mathcal{I}^{p}(S)$.

Proof. (i) Assume $x \succsim^{r} y$. If follows from the definition that $x \in \boldsymbol{c}\{x, y, r\}$. Note that Corollary 15 implies that $x \notin \mathfrak{R}\{x, y, r\}$. If $r$ is a reference in $\{x, y, r\}$ then from Proposition 2 we conclude $x \sim^{p} y$ which completes the proof. If $y$ is a reference in $\{x, y, r\}$ again Proposition 2 implies that $x \succ^{p} y$ in which case the proof is also complete. So assume there are no references in $\{x, y, r\}$. Then $\mathfrak{B}(S)=\{\{x, y\},\{x, r\},\{y, r\}\}$. Since $x \in \boldsymbol{c}\{x, y, r\}$ from TDR we conclude that $x \in \boldsymbol{c}\{x, y\}$ and therefore $x \succsim^{p} y$.

(ii) Take $x, y \in \mathcal{I}^{p}(S)$. Note that since $r$ is a reference in $S$ it follows from Corollary 13 that $r \notin \mathcal{I}^{p}(S)$. Next Proposition 3 implies that $r \notin \boldsymbol{c}\{x, y, r\}$. Since $\boldsymbol{c}$ is non-empty valued we have $x \in \boldsymbol{c}\{x, y, r\}$ or $y \in \boldsymbol{c}\{x, y, r\}$, which in turn imply $x \succsim^{r} y$ or $y \succsim^{r} x$. This completes the proof. 


\subsubsection{The Case of No Maximal Reference}

Lemma 16. Let $\boldsymbol{c}$ be a $R D M$ and $S \subseteq X$. If $\mathfrak{R}_{M}(S)=\varnothing$ then $\boldsymbol{c}(S)=\mathcal{I}^{p}(S)$.

Proof. Assume $\mathfrak{R}_{M}(S)=\varnothing$. We need to show $\boldsymbol{c}(S)=\mathcal{I}^{p}(S)$. To do this first note that Proposition 3 implies $\boldsymbol{c}(S) \subseteq \mathcal{I}^{p}(S)$. So we only need to prove $\mathcal{I}^{p}(S) \subseteq \boldsymbol{c}(S)$. Take $x \in \mathcal{I}^{p}(S)$ and consider the set $\{x, y, z\}$ for two distinct elements $y, z \in S$, both different from $x$.

Step 1: $\mathfrak{R}\{x, y, z\}=\varnothing$.

Proof. By contradiction assume $\mathfrak{R}\{x, y, z\} \neq \varnothing$. First note that by Proposition 6 ii it has to be the case that there is only one reference in $\{x, y, z\}$. Second by Corollary 13 and the fact that $x \in \mathcal{I}^{p}(S)$ we conclude $x \notin \mathfrak{R}(S)$. Wlog, assume $z$ is the reference in $\{x, y, z\}$. Then it has to be the case that

$$
\boldsymbol{c}\{x, y, z\} \subset \boldsymbol{c}\{x, y\}
$$

which implies $\boldsymbol{c}\{x, y\}=\{x, y\}$, which in turn implies $y \succsim^{p} x$. Since $x \in \mathcal{I}^{p}(S)$ PT implies $y \in \mathcal{I}^{p}(S)$. This means $z \in \mathfrak{R}_{M}(S)$ which is not possible. This completes the proof of Step 1 .

Step 2: $x \in \boldsymbol{c}\{x, y, z\}$.

Proof. From Step 1 we have $\mathfrak{R}\{x, y, z\}=\varnothing$. Therefore

$$
\mathfrak{B}\{x, y, z\}=\{\{x, y\},\{y, z\},\{x, z\}\} .
$$

We first argue that $y$ or $z$ can not be the single choice in $\boldsymbol{c}\{x, y, z\}$. To do this, and wlog, assume $\boldsymbol{c}\{x, y, z\}=\{y\}$. Then TDR implies $y \succsim^{p} x$ which in turn means $x, y \in \mathcal{I}^{p}(S)$. Then we conclude that

$$
\boldsymbol{c}\{x, y, z\} \subset \boldsymbol{c}\{x, y\}
$$

which means $z$ is a maximal reference in $S$ which is impossible. Second assume $\{y, z\} \subseteq$ $\boldsymbol{c}\{x, y, z\}$. We show that $x \in \boldsymbol{c}\{x, y, z\}$. From TDR we conclude $y, z \succsim^{p} x$ and, since $x \in \mathcal{I}^{p}(S)$, it follows that $x, y, z \in \mathcal{I}^{p}(S)$ which means $\{x, y, z\}$ is fully indecisive. Using Proposition 6. w we conclude $\boldsymbol{c}\{x, y, z\}=\{x, y, z\}$ which obviously means $x \in \boldsymbol{c}\{x, y, z\}$. Since $\boldsymbol{c}\{x, y, z\} \neq \varnothing$ the proof of this Step 2 is complete.

To finish the proof note that by Lemma 14 ii we conclude $x \in \boldsymbol{c}(S)$. This means $\mathcal{I}^{p}(S) \subseteq$ $\boldsymbol{c}(S)$. Therefore $\boldsymbol{c}(S)=\mathcal{I}^{p}(S)$. 
For the sake of avoiding repetition, I make the the following obvious observations that are used in the remaining argument in several occasions.

Let $\boldsymbol{c}$ be an RDM and $S \subseteq X$. Then

(i) By Corollary 13 for a reference $r \in \mathfrak{R}(S)$, we have $r \notin \mathcal{I}^{p}(S)$. Therefore dropping $r$ from the set $S$ does note change the maximal class of the resulting subset; that is, $\mathcal{I}^{p}(S-r)=\mathcal{I}^{p}(S)$. Note that, using the same argument we deduce that

$$
\mathcal{I}^{p}(S)=\mathcal{I}^{p}(S \backslash R)
$$

for all $R \subseteq \mathfrak{R}(S)$; that is the maximal class of $S$ is the same in all subsets of $S$ which are derived from removing any arbitrary numbers of references from $S$.

(ii) Maximal references in $S-r$ and $S$ only (potentially) differ in $r$. That is $\mathfrak{R}_{M}(S)=$ $\mathfrak{R}_{M}(S-r)$ if $r$ is not a maximal reference in $S$ and $\mathfrak{R}_{M}(S)=\mathfrak{R}_{M}(S-r) \cup\{r\}$ if $r$ is a maximal reference in $S$. Similarly such preservation happens for maximal reference sets of elements; that is for $t \in \mathcal{I}^{p}(S)$, we have $\mathfrak{R}_{M}^{t}(S)=\mathfrak{R}_{M}^{t}(S-r)$ if $r$ is not a maximal reference in $S$ and $\mathfrak{R}_{M}^{t}(S)=\mathfrak{R}_{M}^{t}(S-r) \cup\{r\}$ if $r$ is a maximal reference in $S$.

\subsubsection{Proof of Theorem 2}

Lemma 17. Let c be a $R D M$ and $S \subseteq X$. Also assume $\mathfrak{R}(S)=\mathfrak{R}_{M}(S)=\{r\}$. Then

$$
\boldsymbol{c}(S)=\underset{\mathcal{I}^{p}(S)}{\operatorname{argmax}} \succsim^{r} \cdot 38
$$

\footnotetext{
${ }^{38}$ As discussed before, the domain of relevance for $\succsim^{r}$ is $\mathcal{I}^{p}(S)$; that is, indeed, $\underset{S}{\operatorname{argmax}} \succsim^{r}=\underset{\mathcal{I}^{p}(S)}{\operatorname{argmax}} \succsim^{r}$. To see this note that obviously $\underset{S}{\operatorname{argmax}} \succsim^{r} \subseteq \underset{\mathcal{I}^{p}(S)}{\operatorname{argmax}} \succsim^{r}$. To see the other inclusion take $x \in \underset{\mathcal{I}^{p}(S)}{\operatorname{argmax}} \succsim^{r}$ and $y \in S$ such that $y \notin \mathcal{I}^{p}(S)$. Since $x \in \mathcal{I}^{p}(S)$ this means $x \succ^{p} y$. We must show $x \succsim^{r} y$. First note that by Corollary 13 we conclude that $r \notin \mathcal{I}^{p}(S)$ and since $x \in \mathcal{I}^{p}(S)$ we have $x \succ^{p} r$. Consider the set $\{x, y, r\}$. We must show $x \in \boldsymbol{c}\{x, y, r\}$. Note that $x \in \mathcal{I}^{p}(S)$ implies $x \in \mathcal{I}^{p}\{x, y, r\}$ and by Corollary 13 we conclude $x$ is not a reference in $\{x, y, r\}$. Also note that since there is no pairwise indifference between either $x, y$ or $x, r$ we conclude $y, r$ are also not references in $\{x, y, r\}$. As a result there is no reference in $\{x, y, r\}$ and we conclude

$$
\mathfrak{B}\{x, y, r\}=\{\{x, y\},\{y, r\},\{x, r\}\} .
$$

Since $x \succ^{p} y$ and $x \succ^{p} r$ it follows that $y, r$ are dominated by $x$ in $\{x, y, r\}$ and TDR implies $y, r \notin$ $\boldsymbol{c}\{x, y, r\}$. Finally since $\boldsymbol{c}\{x, y, r\} \neq \varnothing$ we conclude $x \in \boldsymbol{c}\{x, y, r\}$. This in turn means $x \succsim^{r} y$. Therefore, $x \in \underset{S}{\operatorname{argmax}} \succsim^{r}$; that is $\operatorname{argmax} \succsim^{r} \subseteq \operatorname{argmax} \succsim^{r}$. From this we conclude

$$
\underset{\mathcal{I}^{p}(S)}{\operatorname{argmax}} \succsim^{r}=\underset{S}{\operatorname{argmax}} \succsim^{r}
$$
}


Proof. First note that since $r$ is a reference in $S$ it follows that there exists two distinct elements in $S$ both different from $r$ and therefore $|S| \geq 3$.

$(\Leftarrow)$ : Take $x \in \underset{\mathcal{I}^{p}(S)}{\operatorname{argmax}} \succsim^{r}$. Obviously $x \in \mathcal{I}^{p}(S)$. We must show $x \in \boldsymbol{c}(S)$. To do this we first make the following claim.

Claim: For two distinct elements $y, z \in S$, both different from $x$, we have $x \in \boldsymbol{c}\{x, y, z\}$.

Proof. Take two distinct elements $y, z \in S$, both different from $x$, and consider the set $\{x, y, z\}$. We consider two cases here:

\section{Case 1: $\mathfrak{R}\{x, y, z\}=\varnothing$.}

Note that $\mathfrak{R}\{x, y, z\}=\varnothing$ implies $\mathfrak{R}_{M}\{x, y, z\}=\varnothing$. Since $\boldsymbol{c}$ is a RDM on $\{x, y, z\}$ and there are no maximal references in $\{x, y, z\}$ Lemma 16 implies $\boldsymbol{c}\{x, y, z\}=\mathcal{I}^{p}\{x, y, z\}$. Since $x \in \mathcal{I}^{p}(S)$ we conclude $x \in \mathcal{I}^{p}\{x, y, z\}$ and therefore $x \in \boldsymbol{c}\{x, y, z\}$. This completes the proof of this case.

\section{Case 2: $\mathfrak{R}\{x, y, z\} \neq \varnothing$.}

First note that by Proposition 6.ii we conclude $|\Re\{x, y, z\}|=1$. Second Corollary 13 and the fact that $x \in \mathcal{I}^{p}(S)$ imply $x \notin \Re\{x, y, z\}$. Wlog, assume $z \in \mathfrak{R}\{x, y, z\}$. It follows from the definition of references that

$$
\boldsymbol{c}\{x, y, z\} \subset \boldsymbol{c}\{x, y\}
$$

and by Proposition 2 we conclude $y \sim^{p} x$. Since $x \in \mathcal{I}^{p}(S)$ this implies $y \in \mathcal{I}^{p}(S)$. Therefore we conclude $z \in \mathfrak{R}_{M}(S)$ which means $z=r$. Since $x \in \underset{\mathcal{I}^{p}(S)}{\operatorname{argmax}} \succsim^{r}$ we conclude $x \succsim^{r} y$ which in turn implies $x \in \boldsymbol{c}\{x, y, r\}$. This completes the proof in this case and therefore the claim.

To finish the proof of $\Leftarrow$ note that Lemma 14 implies $x \in \boldsymbol{c}(S)$. This finishes the proof of $\Leftarrow$.

$(\Rightarrow)$ : Take $x \in \boldsymbol{c}(S)$. We must show $x \in \underset{\mathcal{I}^{p}(S)}{\operatorname{argmax}} \succsim^{r}$ 
First note that since $r$ is the only reference in $S$ we conclude $\mathfrak{B}(S)=R_{2}(S)$ and that all the elements in $\mathfrak{B}(S)$ are of the form $S-t$ for some $t$ different from $r$. Also note that $r \in A$ for all $A \in \mathfrak{B}(S)$.

We prove this by strong induction on $|S|$. Note that for $|S|=3$ the statement is obvious; that is if $S=\{x, y, r\}$ then if follows from the definition of $\succsim^{r}$ that $x \in c(S)$ if and only if $x \succsim^{r} y$. We base our induction on $|S|=4$.

Induction Base: Assume $|S|=4$. Let $S=\{x, y, z, r\}$. Assume, wlog, that $x \in \boldsymbol{c}(S)$. We need to show $x \in \underset{\mathcal{I}^{p}(S)}{\operatorname{argmax}} \succsim^{r}$. First note that $\mathcal{I}^{p}(S)$

$$
\mathfrak{B}(S)=\{\{x, y, r\},\{x, z, r\},\{y, z, r\}\}
$$

Note that since $r$ is a reference in $S$, Proposition 2 implies that at least two elements in the set $\{x, y, z\}$ are preferred to $r$ under pairwise comparison. Therefore it follows from Proposition 3 that $r \notin \boldsymbol{c}\{x, y, r\}$ and $r \notin \boldsymbol{c}\{y, z, r\}$.

Next note that since $r \notin \mathcal{I}^{p}(S)$ we only need to show $x \succsim^{r} y$ and $x \succsim^{r} z$. To do this, and wlog, consider the set $\{x, y, r\}$. Note that if $x \notin \boldsymbol{c}\{x, y, r\}$ then it has to be the case that $\boldsymbol{c}\{x, y, r\}=\{y\}$. Since $\{x, y, r\}$ is the only element in $\mathfrak{B}(S)$ that contains both $x, y$ this would imply that $y$ dominates $x$ in $S$ and therefore $x \notin \boldsymbol{c}(S)$ which is not possible. So we have $x \in \boldsymbol{c}\{x, y, r\}$. This by definition implies $x \succsim^{r} y$. Therefore $x \in \underset{S}{\operatorname{argmax}} \succsim^{r}=\underset{\mathcal{I}^{r}(S)}{\operatorname{argmax}} \succsim^{r}$. This completes the proof for induction base.

Induction Hypothesis: Assume the statement is true for all sets with cardinality less than or equal to $k$ and let $|S|=k+1$. Take $x \in \boldsymbol{c}(S)$. We first make the following claim.

$\underline{\text { Claim: }} \succsim^{r}$ does not posses any cycles of the length less than or equal to $k-1$ in $\mathcal{I}^{p}(S)$.

Proof. Let $t \leq k-1$. We must show that $\succsim^{r}$ does not possess any cycle of degree $t$ in $\mathcal{I}^{p}(S)$. To do this let $A=\left\{x_{1}, x_{2}, \ldots, x_{t}\right\} \subseteq \mathcal{I}^{p}(S)$ and and assume $x_{1} \succ^{r} x_{2} \succ^{r} \ldots \succ^{r} x_{t}$. We show $x_{1} \succsim^{r} x_{t}$. Consider the set $A \cup\{r\}$. First note that since $\boldsymbol{c}$ is a RDM on $S$ it follows that $\boldsymbol{c}$ is also an RDM in $A \cup\{r\}$. Second, the number of elements in $A, t$, is less than $k-1$ and therefore we have $|A \cup\{r\}|<|S|=k+1$. Third, take an element $x_{i} \in A$. Note that since

$$
x_{i} \succ^{r} x_{i+1} \text { and } x_{i} \sim^{p} x_{i+1}
$$


we conclude $r$ is the unique maximal reference in $A \cup\{r\}$. The last three observations together imply that induction hypothesis is applicable to $A \cup\{r\}$.

To finish the poof of this claim first note that since $r$ is a reference in $A \cup\{r\}$ by Corollary 15 we have $r \notin \boldsymbol{c}(A \cup\{r\})$. Since $\boldsymbol{c}(A \cup\{r\}) \neq \varnothing$ take $x_{i} \in \boldsymbol{c}(S)$. Induction hypothesis implies that $x_{i} \succsim^{r} x_{j}$ for all $j \neq i$. So it has to be the case that $x_{i}=x_{1}$. This means $x_{1} \succsim^{r} x_{t}$. So the proof of the claim is complete.

In order to proof the lemma by contradiction assume $y \succ^{r} x$, for $y \in \mathcal{I}^{p}(S)$. Let

$$
{ }^{x y} \mathfrak{B}(S)=\{A \in \mathfrak{B}(S): x, y \in A\}
$$

that is, the set of all elements of $\mathfrak{B}(S)$ that contain both elements $x, y$.

Next recall that $r \in A$ for all $A \in \mathfrak{B}(S)$ which in turn implies $r \in A$ for all $A \in{ }^{x y} \mathfrak{B}(S)$. Also since $y \succ^{r} x$ and $y \sim^{p} x$ we conclude $r$ is a maximal reference in $A$ for all $A \in{ }^{x y} \mathfrak{B}(S)$. To summarize for all $A \in{ }^{x y} \mathfrak{B}(S)$ we have made the following observations: i. $x, y, r \in A$, ii. $r$ is a maximal reference in $A$. Note that this means induction hypothesis is applicable to all of these sets.

\section{Step 1: ${ }^{x y} \mathfrak{B}(S) \neq \varnothing$.}

Proof. First note that in our induction $|S| \geq 5$ and, therefore, there is at least an element $z \in S$ that is different from $x, y, r$. Since $\mathfrak{B}(S)=R_{2}(S)$ we conclude $S-z$ is an element of $\mathfrak{B}(S)$. Obviously $x, y \in \boldsymbol{c}(S-z)$. This implies $S-z \in{ }^{x y} \mathfrak{B}(S)$ and we conclude ${ }^{x y} \mathfrak{B}(S) \neq \varnothing$.

Step 2: $x, y \notin \boldsymbol{c}(A)$ for all $A \in{ }^{x y} \mathfrak{B}(S)$.

Proof. Take $A \in{ }^{x y} \mathfrak{B}(S)$. Since $y \succ^{r} x$ we conclude $x \notin \underset{\mathcal{I}^{p}(A)}{\operatorname{argmax}} \succsim^{r}$ and therefore induction hypothesis implies that $x \notin \boldsymbol{c}(A)$. Note that since $A$ was an arbitrary element of ${ }^{x y} \mathfrak{B}(S)$ we conclude that $x$ does not beat $y$ in all $A \in{ }^{x y} \mathfrak{B}(S)$.

Next we show that $y$ is also not chosen in all elements of ${ }^{x y} \mathfrak{B}(S)$. To do this take $A \in{ }^{x y} \mathfrak{B}(S)$. If $y \in \boldsymbol{c}(A)$, since $x \notin \boldsymbol{c}(A)$, we conclude that $y$ beats $x$ in $A$. Since $x$ never beats $y$ it follows that $y \vec{S}_{x} x$ and therefore TDR implies $x \notin \boldsymbol{c}(S)$ which is not possible. Since $A$ was arbitrary we conclude $y$ is not chosen in all elements of ${ }^{x y} \mathfrak{B}(S)$ as well. 
Now let $\mathcal{T}=\left\{z \in S: z \in \boldsymbol{c}(A)\right.$ for some $\left.A \in{ }^{x y} \mathfrak{B}(S)\right\}$. First note that since ${ }^{x y} \mathfrak{B}(S) \neq \varnothing$ and also that $\boldsymbol{c}$ is non-empty valued we conclude $\mathcal{T} \neq \varnothing$. Second by Step 2 it follows that $\{x, y\} \cap \mathcal{T}=\varnothing$. Finally since $r$ is a (maximal) reference in all elements of ${ }^{x y} \mathfrak{B}(S)$ Corollary 15 implies that $r$ is never chosen in elements of ${ }^{x y} \mathfrak{B}(S)$ and therefore $r \notin \mathcal{T}$. To summarize we have made the following observation: $\mathcal{T} \cap\{x, y, r\}=\varnothing$.

Step 3: $\mathcal{T} \subseteq \mathcal{I}^{p}(S)$.

Proof. Take $z \in \mathcal{T}$. Then there exists $A \in{ }^{x y} \mathfrak{B}(S)$ such that $z \in \boldsymbol{c}(A)$. Since $x \in A$ we conclude from Proposition 3 that $z \succsim^{p} x$. Next $x \in \mathcal{I}^{p}(S)$ implies $z \in \mathcal{I}^{p}(S)$.

Step 4: For all $z \in \mathcal{T}$ there exists $t \in \mathcal{I}^{p}(S)$ such that $t \succ^{r} z$.

Proof. Take $z \in \mathcal{T}$. Then there exists $A_{1} \in{ }^{x y} \mathfrak{B}(S)$ such that $z \in \boldsymbol{c}\left(A_{1}\right)$. Note that by Step 2 we have $x \notin \boldsymbol{c}\left(A_{1}\right)$, and therefore $z$ beats $x$ in $A_{1}$. Since $x \in \boldsymbol{c}(S)$, TDR implies that $x$ is not dominated by $z$ and therefore it has to be the case that $x$ beats $z_{2}$ in $A_{2}$ for some $A_{2} \in \mathfrak{B}(S) .{ }^{39}$

Next, since $\boldsymbol{c}$ is a RDM in $A_{2}$, and $z \notin \boldsymbol{c}\left(A_{2}\right)$ induction hypothesis implies $z \notin \underset{\mathcal{I}^{p}\left(A_{2}\right)}{\operatorname{argmax}} \succsim^{r}$ and it follows that there exists $t \in \mathcal{I}^{p}\left(A_{2}\right)$ such that $t \succ^{r} z$. To show that $t \in \mathcal{I}^{p}(S)$, note that it follows from the definition of reference relations that $t \in \boldsymbol{c}\{t, z, r\}$. Since $\boldsymbol{c}$ is a RDM on $\{t, z, r\}$ Lemma 14 implies $t \in \mathcal{I}^{p}\{t, z, r\}$. This means $t \succsim^{p} z$. Since $z \in \mathcal{I}^{p}(S)$ we conclude $t \in \mathcal{I}^{p}(S)$.

Step 5: For $z_{1} \in \mathcal{T}$ there exists $z_{2} \in \mathcal{T}$ such that $z_{1} \succ^{r} z_{2}$.

Proof. Take $z_{1} \in \mathcal{T}$. Consider the set $S-z_{1}$. It follows that $S-z_{1} \in \mathfrak{B}(S)$. Note that $z_{1} \notin\{x, y, r\}$. Therefore we conclude $S-z_{1} \in{ }^{x y} \mathfrak{B}(S)$. Take $z_{2} \in \boldsymbol{c}\left(S-z_{1}\right) .{ }^{40}$ Since $S-z_{1} \in{ }^{x y} \mathfrak{B}(S)$ it follows that $z_{2} \in \mathcal{T}$.

Next we show that $z_{1} \succ^{r} z_{2}$. To do this note that induction hypothesis implies $z_{2} \in$ $\operatorname{argmax} \succsim^{r}$ which means $z_{2} \succsim^{r} t$ for all $t \in \mathcal{I}^{p}\left(S-z_{1}\right)$. Also by Step 4 there exists $t^{*} \in \mathcal{I}^{p}(S)$ $\mathcal{I}^{p}\left(S-z_{1}\right)$ such that $t^{*} \succ^{r} z_{2}$. Since $z_{2} \succsim^{r} t$ for all $t \in \mathcal{I}^{p}\left(S-z_{1}\right)$, and also there is an element $t^{*}$ in $\mathcal{I}^{p}(S)$ such that $t^{*} \succ^{r} z_{2}$, there is only one element that can be preferred to $z_{2}$ under $r$ and that is the element which is not in $S-z_{1}: z_{1}$. This means $z_{1} \succ^{r} z_{2}$.

\footnotetext{
${ }^{39}$ In fact, $A_{2}=S-y$.

${ }^{40}$ Note that since $|S| \geq 5$ there exists an element in $S-z_{1}$ different from $x, y, r$ and therefore an element $z_{2}$ exists in $\mathcal{T}$.
} 
To finish the proof of the lemma note that since $x, y \notin \mathcal{T}$ we have $|\mathcal{T}|=t$ for $t \leq k-1$. Take $z_{1} \in \mathcal{T}$ from Step 5 there exists $z_{2} \in \mathcal{T}$ such that $z_{2} \succ^{r} z_{1}$. Applying Step 5 one more time we conclude that there exists $z_{3} \in \mathcal{T}$ such that $z_{3} \succ^{r} z_{2}$. By repeating this argument $t$ times we conclude

$$
z_{1} \succ^{r} z_{2} \succ^{r} \ldots \succ^{r} z_{t} \succ^{r} z_{t+1}
$$

Since $|\mathcal{T}|=t$ this always produces a $\succsim^{r}$-cycle in $\mathcal{I}^{p}(S)$ of degree $t \leq k-1$. This contradicts the starting claim. So proof is complete.

We next prove the following lemma which states this intuitive statement: non-maximal references are irrelevant to RDM's choice behavior.

Lemma 18. Let $\boldsymbol{c}$ be a $R D M$ and $S \subseteq X$. Assume $\mathfrak{R}_{M}(S)=\{r\}$. Also Let $S_{r}$ be the subset of $S$ which is derived by removing all the non-maximal references from $S$. Then

$$
\boldsymbol{c}(S)=\boldsymbol{c}\left(S_{r}\right)
$$

Proof. First note that if $r$ is the only reference in $S$; that is if $|\mathfrak{R}(S)|=1$ then $S_{r}=S$ and the statement is obvious. So for the rest of this argument assume $|\mathfrak{R}(S)| \geq 2$. Note that this latter fact implies $\mathfrak{B}(S)=R_{1}(S)$. Therefore elements of $\mathfrak{B}(S)$ are derive by taking references out of the set $S$. We start with the following tow claims.

Claim 1: If $x \in \boldsymbol{c}(S)$ then there exists a non-maximal reference $t$ such that $x \in \boldsymbol{c}(S-t)$. Proof. Take $x \in \boldsymbol{c}(S)$. First note that $\mathcal{I}^{p}(S-r)=\mathcal{I}^{p}(S)$. Since $S-r$ does note posses any maximal reference Lemma 16 implies $\boldsymbol{c}(S-r)=\mathcal{I}^{p}(S-r)=\mathcal{I}^{p}(S)$. This means $x$ does not beat any elements of $\mathcal{I}^{p}(S)$ in $S-r$. Now take a reference $t_{1} \neq r$ in $S$ and consider the set $S-t_{1}$. If $x \in \boldsymbol{c}\left(S-t_{1}\right)$ there is nothing to prove. So assume $x \notin \boldsymbol{c}\left(S-t_{1}\right)$ and take $y \in \boldsymbol{c}\left(S-t_{1}\right)$. It follows that $y$ beats $x$ in $S-t_{1}$. Since $x \in \boldsymbol{c}(S)$, TDR implies that $x$ is not dominated by $y$ and therefore there exists a reference $t_{2}$ such that $x$ beats $y$ in $S-t_{2}$. Note that since $x$ is beating $y$ in $S-t_{2}$ we conclude $t_{2} \neq r$ and therefore $t_{2}$ is a non-maximal reference. This finishes the proof of this claim.

Claim 2: If $x \notin \boldsymbol{c}(S)$ then there exists a non-maximal reference $t \neq r$ in $S$ such that $x \notin \boldsymbol{c}(S-t)$.

Proof. First note that $|S| \geq 4{ }^{41}$ Recall from the proof of Claim 1 that $\boldsymbol{c}(S-r)=\mathcal{I}^{p}(S)$.

\footnotetext{
${ }^{41}$ To see this note that since $r$ is a maximal reference in $S$ there exists, at least two distinct elements, $t_{1}, t_{2}$ in $\mathcal{I}^{p}(S)$, both different from $r$. Next since $|\mathfrak{R}(S)| \geq 2$ there exists a reference $s \neq r$ in $S$. Since $s$ is a
} 
We consider two cases here.

Case 1: $x \notin \boldsymbol{c}(S-r)$.

Since $\boldsymbol{c}(S-r)=\mathcal{I}^{p}(S)$ this means that $x \notin \mathcal{I}^{p}(S)$. Now consider the set $S-t$ for a reference $t \neq r$ in $S$. Proposition 3 implies that $\boldsymbol{c}(S-t) \subseteq \mathcal{I}^{p}(S-t)=\mathcal{I}^{p}(S)$ and therefore we conclude $x \notin \boldsymbol{c}(S-t)$. This completes the proof in this case.

Case 2: $x \in \boldsymbol{c}(S-r)$.

First from $|S| \geq 4$ it follows that $S$ satisfies BUR. Since $x \notin \boldsymbol{c}(S)$ we conclude that there exists $y \in S$ such that $y$ dominates $x$ in $S$. Therefore there exists a reference $t$ in $S$ such that $y, x \in \boldsymbol{c}(S-t)$ and $y$ is chosen in $S-t$ and $x$ is not chosen in $S-t$. So it only remains to prove that $t \neq r$. This follows from the fact that $x \in \boldsymbol{c}(S-r)$; that is $y$ does not beat $x$ in $S-r$. This completes the proof in this case.

Now we proceed to prove the Lemma.

First we show $\boldsymbol{c}(S) \subseteq \boldsymbol{c}\left(S_{r}\right)$. To do this take $x \in \boldsymbol{c}(S)$. By Claim 1 there exists a nonmaximal reference $t_{1} \neq r$ in $S$ such that $x \in \boldsymbol{c}(S-t)$. If $S-t=S_{r}$ the proof is complete. If not then there must exist another reference different from $r$ in $S$ and therefore $|\mathfrak{R}(S-t)| \geq 2$. Applying Claim 1 one more time we conclude there exits a non-maximal reference $t_{2}$ in $S$ such that $x \in \boldsymbol{c}\left(\left(S-t_{1}\right)-t_{2}\right)$. By repeating this algorithm for a finite number of time, and after taking all non-maximal references out of $S$, we conclude $x \in \boldsymbol{c}\left(S_{r}\right)$. This means $\boldsymbol{c}(S) \subseteq \boldsymbol{c}\left(S_{r}\right)$.

To show $\boldsymbol{c}\left(S_{r}\right) \subseteq \boldsymbol{c}(S)$ we prove that $x \notin \boldsymbol{c}(S) \Longrightarrow x \notin \boldsymbol{c}\left(S_{r}\right)$. Assume $x \notin \boldsymbol{c}(S)$. By Claim 2 there exists a non-maximal reference $t_{1} \neq r$ in $S$ such that $x \notin \boldsymbol{c}(S-t)$. If $S-t=S_{r}$ the proof is complete. If not then there must exist another reference different from $r$ in $S$ and therefore $|\Re(S-t)| \geq 2$. Applying Claim 2 one more time we conclude there exits a non-maximal reference $t_{2}$ in $S$ such that $x \notin \boldsymbol{c}\left(\left(S-t_{1}\right)-t_{2}\right)$. By repeating this algorithm for a finite number of time, and after taking all non-maximal references out of $S$, we conclude $x \notin \boldsymbol{c}\left(S_{r}\right)$. This implies $\boldsymbol{c}\left(S_{r}\right) \subseteq \boldsymbol{c}(S)$, and therefore $\boldsymbol{c}(S)=\boldsymbol{c}\left(S_{r}\right)$.

Lemma 19. Let $\boldsymbol{c}$ be a $R D M$ such that $\mathfrak{R}_{M}(S)=\{r\}$. Then

$$
c(S)=\underset{\mathcal{I}^{p}(S)}{\operatorname{argmax}} \succsim^{r}
$$

reference in $S$ it follows from Proposition 6, iv that $s \notin \mathcal{I}^{p}(S)$. This in turn means $t_{1}, t_{2} \succ^{p} s$ and there for $t_{1}, t_{2}$ are different from $s$. Therefore we conclude $t_{1}, t_{2}, r, s$ are four distinct elements. This means $|S| \geq 4$. 
Proof. Let $S_{r}$ be the subset of $S$ which is derived by taking all the non-maximal references out of $S$. Note that $\mathcal{I}^{p}\left(S_{r}\right)=\mathcal{I}^{p}(S)$. It follows that $\boldsymbol{c}$ is a RDM on $S_{r}$ and $S_{r}$ only possesses one reference, $r$, which is maximal. Then from Lemmas 18 , and 17 we conclude

$$
x \in \boldsymbol{c}(S) \stackrel{\operatorname{Lem}[18}{\Longleftrightarrow} x \in \boldsymbol{c}\left(S_{r}\right) \stackrel{\operatorname{Lem}[17}{\Longleftrightarrow} x \in \underset{\mathcal{I}^{p}\left(S_{r}\right)}{\operatorname{argmax}} \succsim^{r}=\underset{\mathcal{I}^{p}(S)}{\operatorname{argmax}} \succsim^{r} .
$$

Therefore the proof is complete.

Theorem 2, (Acyclicity of Reference Relations) Let $\boldsymbol{c}$ be a $R D M$ and $r \in \mathfrak{R}(S)$. $\succsim^{r}$ defines an acyclic relation on $\mathcal{I}^{p}(S)$.

Proof. Let $t \leq\left|\mathcal{I}^{p}(S)\right|$. We must show that $r$ does not possess any cycle of degree $t$. To do this let $A=\left\{x_{1}, x_{2}, \ldots, x_{t}\right\} \subseteq \mathcal{I}^{p}(S)$ and assume $x_{1} \succ^{r} x_{2} \succ^{r} \ldots \succ^{r} x_{t}$. We show $x_{1} \succsim^{r} x_{t}$. Consider the set $A \cup\{r\}$. First assume that $r$ is not a maximal reference in $A \cup\{r\}$ and consider the set $\left\{x_{1}, x_{t}, r\right\}$. Note that since $x_{1} \succ^{p} r$ Proposition 3 implies that $r \notin \boldsymbol{c}\left\{x_{1}, x_{2}, r\right\}$. Since $r$ is not a maximal reference in $A \cup\{r\}$ then it should be the case that $\boldsymbol{c}\left\{x_{1}, x_{t}, r\right\}=\boldsymbol{c}\left\{x_{1}, x_{t}\right\}=\left\{x_{1}, x_{t}\right\}$, which, in turn, means $x_{1} \succsim^{r} x_{t}$. To finish the proof assume that $r$ is a maximal reference in $A \cup\{r\}$. First note that $\mathcal{I}^{p}(A \cup\{r\})=A$. Second, since $r \notin \boldsymbol{c}(A \cup\{r\})$ and $\boldsymbol{c}(A \cup\{r\}) \neq \varnothing$ take $x_{i} \in \boldsymbol{c}(A \cup\{r\})$. Then Lemma 19 implies that $x_{i} \in \underset{a}{\operatorname{argmax}} \succsim^{r}$; that is $x_{i} \succsim^{r} x_{j}$. Therefore $x_{i}=x_{1}$ and as a result $x_{1} \succsim^{r} x_{t}$. This completes the proof. ${ }^{42}$

This next direct corollary of Theorem 2 is useful in the proof of the final theorem.

Corollary 20. Let $\boldsymbol{c}$ be a $R D M$ and $S \subseteq X$. For a maximal reference $r$ is $S$ we have $\underset{I^{p}(S)}{\operatorname{argmax}} \succsim^{r} \neq \varnothing$. $\mathcal{I}^{p}(S)$

Proof. From Proposition 4 .ii and Theorem 2 we conclude $\succsim^{r}$ defines a complete and acylice relation on $\mathcal{I}^{p}(S)$. Therefore $\succsim^{r}$ attains a maximum on $\mathcal{I}^{p}(S)$ and we conclude $\underset{\mathcal{I}^{p}(S)}{\operatorname{argmax}} \succsim^{r} \neq$ $\varnothing$.

\footnotetext{
${ }^{42}$ It worths noting that $\succsim^{r}$, indeed, defines an acyclic relation on $S$. To see this assume, for $x_{i} \in S$, $x_{1} \succ^{r} x_{2} \succ^{r} \ldots \succ^{r} x_{n}$. Let $A=\left\{x_{1}, x_{2}, \ldots, x_{n}\right\} \cup\{r\}$. Note that $x_{1} \in \operatorname{argmax} \succsim^{r}$ we conclude that $x_{1} \in \boldsymbol{c}(A)$ which in turn, using Proposition 3 implies $x_{1} \in \mathcal{I}^{p}(A)$. If $x_{n} \in \mathcal{I}^{p}(A)$ then Proposition 4 i implies that $x_{n-1} \in \mathcal{I}^{p}(A)$ which in turn, using the same proposition, implies $x_{i} \in \mathcal{I}^{p}(A)$ for all $i \in\{1,2, \ldots, n\}$. Therefore from Theorem 2 we conclude $x_{1} \succsim^{r} x_{n}$ and the proof is complete. If, otherwise, $x_{n} \notin \mathcal{I}^{p}(S)$ then we have $x_{1} \succ^{p} x_{n}$ and Proposition 4 i implies that $x_{n} \succsim^{r} x_{1}$ can not hold and therefore $x_{n} \notin \boldsymbol{c}\left\{x_{1}, x_{n}, r\right\}$. Since, $r \notin \boldsymbol{c}\left\{x_{1}, x_{n}, r\right\}$ we conclude $x_{1} \in \boldsymbol{c}\left\{x_{1}, x_{n}, r\right\}$ and therefore $x_{1} \succsim^{r} x_{n}$.
} 


\subsubsection{Proofs of Lemmas 9,10}

Lemma 9. Let $\boldsymbol{c}$ be a $R D M$ and $S \subseteq X$ such that $|\Re(S)| \geq 2$. If $x \in \boldsymbol{c}(S)$ then there exists a reference $r$ in $S$ such that $x \in \boldsymbol{c}(S-r)$.

Proof. Assume $|\mathfrak{R}(S)| \geq 2$. Note that all elements of $\mathfrak{B}(S)$ are of the form $S-r$ for some references in $r$ in $S$. Now take $S-r_{1}$ in $\mathfrak{R}(S)$. If $x \in \boldsymbol{c}\left(S-r_{1}\right)$ then there is nothing to prove. So take $y$ different from $x$ such that $y \in \boldsymbol{c}\left(S-r_{1}\right)$. If follows that $y$ beats $x$ in $S-r_{1}$. Since $x \in \boldsymbol{c}(S)$ and $\boldsymbol{c}$ satisfies TDR we conclude there exists $r_{2} \in \mathfrak{R}(S)$ such that $x$ beats $y$ in $S-r_{2} \cdot{ }^{43}$ In particular, we have $x \in \boldsymbol{c}\left(S-r_{2}\right)$.

Lemma 10. Let $\boldsymbol{c}$ be a $R D M$ and $S \subseteq X$ that possesses at least two maximal references. For any reference $r$ in $S$ assume

$$
t^{*} \in \boldsymbol{c}(S-r) \Longleftrightarrow\left|\mathfrak{R}_{M}^{t^{*}}(S-r)\right| \geq\left|\mathfrak{R}_{M}^{t}(S-r)\right| \text {, for all } t \in \mathcal{I}^{p}(S-r .)
$$

Also assume that $x \in \boldsymbol{c}(S)$ and $x, y \in \boldsymbol{c}(S-\bar{r})$ for a reference $\bar{r}$ in $S$. If $\bar{r}$ refers to $y$ then $\bar{r}$ refers to $x$.

Proof. To start we partition the set $\mathcal{I}^{p}(S)$ into the following sets:

$$
\begin{gathered}
\mathcal{T}_{0}=\left\{t \in \mathcal{I}^{p}(S): t \notin \boldsymbol{c}(S-\bar{r})\right\}, \\
\mathcal{T}_{1}=\{t \in \boldsymbol{c}(S-\bar{r}): \bar{r} \text { does not refer to } t\}, \\
\mathcal{T}_{2}=\{t \in \boldsymbol{c}(S-\bar{r}): \bar{r} \text { refers to } t\} .
\end{gathered}
$$

Note that by Corollary $15 r \notin \mathcal{I}^{p}(S)$ and, as a result, $\left\{\mathcal{T}_{0}, \mathcal{T}_{1}, \mathcal{T}_{2}\right\}$ partitions the set $\mathcal{I}^{p}(S)$.

Assume $\bar{r}$ refers to $y$. Note that by definition $\bar{r}$ is a maximal reference. Also note that since $y \in \boldsymbol{c}(S-\bar{r})$ it follows that $y \in \mathcal{T}_{2}$ and therefore $\mathcal{T}_{2} \neq \varnothing$. By contradiction assume that $\bar{r}$ does not refer to $x$. Note that this implies $x \in \mathcal{T}_{1}$.

Step 1:

$$
\left|\Re_{M}^{z}(S)\right|>\left|\Re_{M}^{x}(S)\right|
$$

for all $z \in \mathcal{T}_{2}$.

\footnotetext{
${ }^{43}$ Note that such $r_{2}$ exists since $|\mathfrak{R}(S)| \geq 2$.
} 
Proof. Take an arbitrary element $z \in \mathcal{T}_{2}$. We have $x, z$ are chosen in $S-\bar{r}$ and therefore

$$
\left|\mathfrak{R}_{M}^{x}(S-\bar{r})\right|=\left|\mathfrak{R}_{M}^{z}(S-\bar{r})\right|
$$

Since $\bar{r}$ refers to $z$ and not to $x$, adding it to set the set $S-\bar{r}$ increases the number of $z$-maximal references over $x$-maximal references; that is

$$
\left|\Re_{M}^{z}(S)\right|>\left|\Re_{M}^{x}(S)\right|
$$

Step 2: $x$ does note beat $z$ in $A$, for all $A \in \mathfrak{B}(S)$, and all $z \in \mathcal{T}_{2}$.

Proof. First note that since $|\mathfrak{R}(S)| \geq\left|\mathfrak{R}_{M}(S)\right| \geq 2$ then all $A \in \mathfrak{B}(S)$ are of the form $S-r$ for some reference $r$ in $S$. So take an arbitrary element $A=S-r \in \mathfrak{B}(S)$. Also take an arbitrary element $z \in \mathcal{T}_{2}$. From (1), and in $S$, the number of $z$-maximal references is more than the number of $x$-maximal references, and therefore dropping $r$ will in the worse case equalize these two numbers (that worse case happens when $r$ refers to $z$ and not $x$ ). Therefore

$$
\left|\Re_{M}^{z}(S-r)\right| \geq\left|\Re_{M}^{x}(S-r)\right|
$$

To complete the proof of this step we show that $x \in \boldsymbol{c}(S-r)$ implies $z \in \boldsymbol{c}(S-r)$ and therefore $x$ does not beat $z$ in $S-r$. In order to do this assume $x \in \boldsymbol{c}(S-r)$. Then it follows that

$$
\left|\mathfrak{R}_{M}^{x}(S-r)\right| \geq\left|\mathfrak{R}_{M}^{t}(S-r)\right|,
$$

for all $t \in \mathcal{I}^{p}(S-r)$. From (2) and (3) we conclude

$$
\left|\mathfrak{R}_{M}^{z}(S-r)\right| \geq\left|\mathfrak{R}_{M}^{t}(S-r)\right|
$$

for all $t \in \mathcal{I}^{p}(S-r)$. This implies $z \in \boldsymbol{c}(S-r)$. Therefore we conclude $x$ does not beat $z$ in $S-r$.

$\underline{\text { Step 3: }}$ There exists a $x$-maximal reference in $S$.

Proof. Since there are at least two maximal references in $S$ we conclude that $S-\bar{r}$ has at least one maximal reference. Take $r \in \mathfrak{R}_{M}(S-\bar{r})$, By Corollary 20 we conclude $\underset{\mathcal{I}^{p}(S-\bar{r})}{\operatorname{argmax}} \succsim^{r} \neq \varnothing$ 
and therefore $r$ refers to an element of $\mathcal{I}^{p}(S-\bar{r})$; that is each maximal references in the set $S-\bar{r}$ refers, at least, to one element of $\mathcal{I}^{p}(S-\bar{r})$. Since $x \in \boldsymbol{c}(S-\bar{r})$ it follows that, in $S-\bar{r}$, the number of $x$-maximal references is greater than or equal to the number of $t$-maximal references, for all $t \in \mathcal{I}^{p}(S-\bar{r})$. Since there exists at least one maximal references in $S-\bar{r}$ if $x$ has no maximal reference in $S-r$ this last assertion would be violated. Therefore there exists a $x$-maximal reference in $S-r$.

As a result of Step 3 let $r_{x}$ be a (maximal) reference in $S$ that refers to $x$.

Step 4: $x \notin \boldsymbol{c}\left(S-r_{x}\right)$.

Proof. Take $z \in \mathcal{T}_{2}$. Recall from (1) that $z$ has more maximal references in $S$ than $x$. Since $r_{x}$ refers to $x$ dropping $r_{x}$ from $S$ results in

$$
\left|\mathfrak{R}_{M}^{z}\left(S-r_{x}\right)\right|>\left|\mathfrak{R}_{M}^{x}\left(S-r_{x}\right)\right|
$$

It follows that $x \notin \boldsymbol{c}\left(S-r_{x}\right)$.

Step 5:

$$
\left|\mathfrak{R}_{M}^{z}\left(S-r_{x}\right)\right| \geq\left|\mathfrak{R}_{M}^{t}\left(S-r_{x}\right)\right|
$$

for all $z \in \mathcal{T}_{2}$, and all $t \in \mathcal{T}_{0}$.

Proof. Take an arbitrary element $t \in \mathcal{T}_{0}$ and an arbitrary element $z \in \mathcal{T}_{2}$. First note that since $\bar{r}$ refers to $z$ (and $r_{x}$ might or might not refer to $z$ ) then dropping $\bar{r}$ from $S$ does not increase the number of maximal references of $z$ relative to dropping $r_{x}$. Therefore we have

$$
\left|\mathfrak{R}_{M}^{z}\left(S-r_{x}\right)\right| \geq\left|\Re_{M}^{z}(S-\bar{r})\right|
$$

Next recall that we have i. $z$ is chosen in $S-\bar{r}$ and ii. $t$ is not chosen in $S-\bar{r}$. Therefore we conclude

$$
\left|\mathfrak{R}_{M}^{z}(S-\bar{r})\right|>\left|\mathfrak{R}_{M}^{t}(S-\bar{r})\right|\left(\text { or equivalently }\left|\mathfrak{R}_{M}^{z}(S-\bar{r})\right| \geq\left|\mathfrak{R}_{M}^{t}(S-\bar{r})\right|+1\right)
$$

Finally consider the two sets $S-r_{x}$, and $S-\bar{r}$. Since these sets are derived by dropping only of reference from the set $S$ it is true that the difference between the number of $t$-maximal references in these two sets is less then or equal to 1 ; that is 


$$
\left|\mathfrak{R}_{M}^{t}(S-\bar{r})\right|+1 \geq\left|\mathfrak{R}_{M}^{t}\left(S-r_{x}\right)\right|
$$

Combining these assertion in order we conclude

$$
\left|\mathfrak{R}_{M}^{z}\left(S-r_{x}\right)\right| \geq\left|\mathfrak{R}_{M}^{z}(S-\bar{r})\right| \geq\left|\mathfrak{R}_{M}^{t}(S-\bar{r})\right|+1 \geq\left|\mathfrak{R}_{M}^{t}\left(S-r_{x}\right)\right| .
$$

This complete the proof of this step.

Step 6: for $z \in \mathcal{T}_{2}$ and $t \in \mathcal{T}_{1}$ we have:

$$
\left|\mathfrak{R}_{M}^{z}\left(S-r_{x}\right)\right| \geq\left|\mathfrak{R}_{M}^{t}\left(S-r_{x}\right)\right|
$$

Proof. Take an arbitrary element $z \in \mathcal{T}_{2}$ and an arbitrary element $t \in \mathcal{T}_{1}$. Note that since $\bar{r}$ refers to $z$ (and $r_{x}$ might or might not refer to $z$ ) then dropping $\bar{r}$ from $S$ does not increase the number of maximal references of $z$ relative to dropping $r_{x}$. Therefore we have

$$
\left|\mathfrak{R}_{M}^{z}\left(S-r_{x}\right)\right| \geq\left|\mathfrak{R}_{M}^{z}(S-\bar{r})\right|
$$

Next recall $z, t$ are chosen in $S-\bar{r}$. Therefore it follows that

$$
\left|\mathfrak{R}_{M}^{z}(S-\bar{r})\right|=\left|\mathfrak{R}_{M}^{t}(S-\bar{r})\right|
$$

Combining these two assertions then we conclude

$$
\left|\mathfrak{R}_{M}^{z}\left(S-r_{x}\right)\right| \geq\left|\mathfrak{R}_{M}^{z}(S-\bar{r})\right|=\left|\mathfrak{R}_{M}^{t}\left(S-r_{x}\right)\right|
$$

This complete the proof of this claim.

Step 7: There exists $z^{*} \in \mathcal{T}_{2}$ such that $z^{*} \in \boldsymbol{c}\left(S-r_{x}\right)$.

Proof. Take $z \in \boldsymbol{c}\left(S-r_{x}\right)$. First note that this implies Then

$$
\left|\mathfrak{R}_{M}^{z}\left(S-r_{x}\right)\right| \geq\left|\mathfrak{R}_{M}^{t}\left(S-r_{x}\right)\right|
$$

for all $t \in \mathcal{I}^{p}\left(S-r_{x}\right)$. Second Proposition 3 implies that $\boldsymbol{c}\left(S-r_{x}\right) \subseteq \mathcal{I}^{p}\left(S-r_{x}\right)=\mathcal{I}^{p}(S)$ and we conclude $z \in \mathcal{I}^{p}(S)$. Recall that $\left\{\mathcal{T}_{0}, \mathcal{T}_{1}, \mathcal{T}_{2}\right\}$ partitions the set $\mathcal{I}^{p}(S)$. If $z \in \mathcal{T}_{2}$ there is nothing to prove. So assume $z$ in either $\mathcal{T}_{0}$ or $\mathcal{T}_{1}$; that is $z \in \mathcal{T}_{0} \cup \mathcal{T}_{1}$. Next take $z^{*} \in \mathcal{T}_{2}$. By Steps 5,6 we conclude

$$
\left|\mathfrak{R}_{M}^{z^{*}}\left(S-r_{x}\right)\right| \geq\left|\mathfrak{R}_{M}^{z}\left(S-r_{x}\right)\right|
$$


Combining the last two equations we conclude

$$
\left|\mathfrak{R}_{M}^{z^{*}}\left(S-r_{x}\right)\right| \geq\left|\mathfrak{R}_{M}^{t}\left(S-r_{x}\right)\right|
$$

for all $t \in \mathcal{I}^{p}\left(S-r_{x}\right)$. It follows that $z^{*} \in \boldsymbol{c}\left(S-r_{x}\right)$. This completes the proof of this step.

To finish the proof of the Lemma note that by Step 7 there exists $z^{*} \in \mathcal{T}_{2}$ such that $z^{*} \in \boldsymbol{c}\left(S-r_{x}\right)$. Also from Step 4 we have $x \notin \boldsymbol{c}\left(S-r_{x}\right)$. This means $z^{*}$ beats $x$ in $S-r_{x}$.

Next from Step 2 we have $x$ does not beat $z^{*}$ in all $A \in \mathfrak{B}(S)$. This implies $z^{*} \vec{S}_{x}$. This means $x$ is not a maximal element of $\underset{S}{\vec{b}}$, which in turn by TDR, implies $x \notin \boldsymbol{c}(S)$. A contradiction.

\subsection{Independence of Axioms}

In this section I show the independence of TDR, BUR, and PT using the following examples (circles represent choice). For the following three examples let $S=\{x, y, z\}$.

Example 1. Let $\boldsymbol{c}_{1}$ be the following choice correspondence:

$$
\begin{gathered}
\{(y, y\} \\
\{x, y\},\{(y, z\},\{(x, z\}
\end{gathered}
$$

There are no reference in $S$ under $\boldsymbol{c}_{1}$. Therefore

$$
\mathfrak{B}(S)=\{\{x, y\},\{y, z\},\{x, z\}\}
$$

$\boldsymbol{c}_{1}$, obviously, satisfies PT. Also since $x$ is the unique maximal element of $\boldsymbol{}_{S}$ and $x \in \boldsymbol{c}(S)$ we conclude that $\boldsymbol{c}_{1}$ also satisfies BUR. However, it does not satisfy TDR. In particular, $y \in$ $\boldsymbol{c}(S)$ but $x \triangleright_{S} y$. $\boldsymbol{c}_{1}$ does not satisfy WARP, because $x, z \in\{x, z\} \cap\{x, y, z\}, z \in \boldsymbol{c}\{x, y, z\}$, and $x \in \boldsymbol{c}\{x, z\}$, but $z \notin \boldsymbol{c}\{x, z\}$.

Example 2. Let $\boldsymbol{c}_{2}$ be the following choice correspondence:

$$
\begin{gathered}
\{(x, y, z\} \\
\{(y, y\},\{y, z\},\{x, z\}
\end{gathered}
$$

This is an example of attraction effect (or status quo bias) discussed in the paper. $z$ is the unique reference in $S$ under $\boldsymbol{c}_{2}$. Therefore

$$
\mathfrak{B}(S)=\{\{y, z\},\{x, z\}\} .
$$


Obviously $\boldsymbol{c}_{1}$ satisfies PT. Since $\{x\}=\boldsymbol{c}(S)$ and $x$ is not dominated by $y$, nor is it dominated by $z$, then $\boldsymbol{c}_{2}$ satisfies TDR. $\boldsymbol{c}_{2}$, however, does not satisfy BUR. To see this note that $y$ is not dominated by $x$ nor $z$ and therefore is a maximal element of $\vec{S}_{S}$, but not chosen in $S$. Note that $\boldsymbol{c}_{2}$ is a RDM. Finally $\boldsymbol{c}_{2}$ does not satisfy WARP since $x, y \in\{x, y\} \cap\{x, y, z\}$, and $y \in \boldsymbol{c}\{x, y\}$, and $x \in \boldsymbol{c}\{x, y, z\}$, but $y \notin \boldsymbol{c}\{x, y, z\}$.

Example 3. Let $\boldsymbol{c}_{3}$ be the following choice correspondence:

$$
\begin{gathered}
\{(x, y, z\} \\
\{x, y\},\{y, z\},\{x, z\}
\end{gathered}
$$

$z$ is the unique reference in $S$ under $c_{3}$. Therefore

$$
\mathfrak{B}(S)=\{\{y, z\},\{x, z\}\}
$$

$x$ is the unique maximal element of ${ }_{S}$ which is chosen in $S$. Therefore $\boldsymbol{c}_{3}$ satisfies both TDR and BUR. However, it violates PT and, as a result, WARP.

The following table summarizes the aforementioned argument:

\begin{tabular}{|c|c|c|c|c|}
\hline & TDR & BUR & PT & WARP \\
\hline $\boldsymbol{c}_{1}$ & $\times$ & $\checkmark$ & $\checkmark$ & $\times$ \\
\hline $\boldsymbol{c}_{2}$ & $\checkmark$ & $\times$ & $\checkmark$ & $\times$ \\
\hline $\boldsymbol{c}_{3}$ & $\checkmark$ & $\checkmark$ & $\times$ & $\times$ \\
\hline
\end{tabular}

\subsection{RDM: Majority Rule Vs. Condorcet Criterion}

The notion of RDM can be conveniently perceived as a voting situation where candidate are represented by the elements of the most preferred class, $\mathcal{I}^{p}(S)$, and voters are references whose rankings of the candidates are given with the associated reference preference. In the view of the result in Theorem 3 , then, RDM is consistent with the majority rule; that is: the candidate who has the majority of the votes is elected. RDM, however, is not consistent with the Condorcet criterion. To see this let $S=\left\{x_{1}, x_{2}, x_{3}, x_{4}, r_{1}, r_{2}, r_{3}\right\}$, where $r_{i}$ is a reference (voters), and $\left\{x_{1}, x_{2}, x_{3}, x_{4}\right\}=\mathcal{I}^{p}(S)$ (candidates). Assume the following preference for each voter:

$$
\begin{aligned}
& r_{1}: x_{1} \succ^{r_{1}} x_{2} \succ^{r_{1}} x_{3} \succ^{r_{1}} x_{4} \\
& r_{2}: x_{2} \succ^{r_{2}} x_{3} \succ^{r_{2}} x_{4} \succ^{r_{2}} x_{1}
\end{aligned}
$$




$$
r_{3}: x_{3} \succ^{r_{3}} x_{2} \succ^{r_{3}} x_{4} \succ^{r_{3}} x_{1}
$$

From Theorem 3 it follows that $\boldsymbol{c}(S)=\left\{x_{1}, x_{2}, x_{3}\right\}$. However, $x_{1}$ does not satisfy the Condorcet criterion. In particular, if restricted to only $x_{1}, x_{4}$, the latter has more votes than the former.

\section{References}

Apesteguia, J. and M. A. Ballester (2013). Choice by sequential procedures. Games and Econoimcs Behavior 77, 90-99.

Arrow, K. J. (1959). Rational choice function and orderings. Economica 26(102), 121127.

Bordalo, P., N. Gennaioli, and A. Shleifer (2013). Salience and consumer choice. Journal of Political Economy 121 (5), 803-843.

Carbajal, J. C. and J. C. Ely (2016). A model of price discrimination under loss aversion and state-contingent reference points. Theoretical Economics 11, 455-485.

Cherepanov, V., T. Feddersen, and A. Sandroni (2013). Rationalization. Theoretical Economics 8, 775-800.

de Clippel, G. and K. Eliaz (2012). Reason-based choice: A bargaining rational for the attraction and compromise effect. Theoretical Economics 7, 125-162.

Doyl, J. R., D. J. O'Conner, G. M. Reynolds, and P. A. Bottomly (1999). The robustness of the assymetrically dominated effect: Buying frames, phantom alternatives, and in-store purchases. Psychology and Marketing 36(3), 225243.

Edgeworth, F. Y. (1881). Mathematical psychics: An essay on the application of mathematics to the moral sciences, Volume 10. Kegan Paul.

Gerasimou, G. (2016). Partially dominant choice. Econ Theory 61, 127-145.

Hardie, B. G., E. J. Johnson, and P. S. Fader (1993). Model of loss aversion and reference dependece effects on brand choice. Marketing Science 12(4), 378-394.

Huber, J., J. W. Payne, and C. Puto (1982). Adding asymmetrically dominated alternatives: Violation of regularity and the similarity hypothesis. Journal of Consumer Research 9(1), 90-98.

Kahneman, D. and A. Tversky (1979). Prospect theory: An analysis of decision under risk. Econometrica 47(2), 263-292.

Kahneman, D. and A. Tversky (1984). Choice, values, and frames. American Pscyhologist $X X X I X, 341-350$.

Kalai, G., A. Rubinstein, and R. Spiegler (2002). Rationalizing choice functions by multiple rationales. Econometrica $70(6), 2481-2488$. 
Kamenica, E. (2008). Contextual inference in markets: On the informational content of product lines. Econometrica 98(5), 2127-2149.

Knetsch, J. L. (1989). The endowment effect and evidence of nonreversible indifference curves. The American Economics Review 79(5), 1277-1284.

Knetsch, J. L. and J. A. Sinden (1984). Willingness to pay and compensation demanded: Experimental evidence of an unexpected disparity in measures of value. The Quarterly Journal of Economics 99(3), 507-521.

Köszegi, B. and M. Rabin (2006). A model of reference-dependent preferences. The Quarterly Journal of Economics 121( 4), 1133-1165.

Kőszegi, B. and M. Rabin (2007). Reference-dependent risk attitudes. The American Economic Review 97(4), 1047-1073.

Lleras, J. S., Y. Masatlioglu, D. Nakajima, and E. Ozbay (2015). When more is less: Limited consideration. Unpublished Paper.

Lombardi, M. (2009). Reason-based choice correspondences. Mathematical Social Science 57, $58-66$.

Manzini, P. and M. Mariotti (2007). Sequentially rationalizable choice. The American Economic Review 97(5), 1824-1839.

Manzini, P. and M. Mariotti (2010). Revealed preference theory and boundedly rational choice procedures: An experiment. Unpublished Paper.

Marshall, A. (1890). Principles of economics: An introductory volume. Macmillan London.

Mas-Collel, A., M. D. Whinston, and J. R. Green (1995). Microeconomic Theory. 198 Madison Avenue, New York, New York, 10016: Oxford University Press, Inc.

Masatlioglu, Y., D. Nakajima, and E. Y. Ozbay (2012). Reveald attention. The American Economics Review 102(5), 2183-2205.

Masatlioglu, Y. and E. A. Ok (2005). Rational choice with status quo bias. Journal of Economics Theory 121, 1-29.

Ok, E. A., P. Ortovela, and G. Riella (2011). Theory of product differentiation in the presence of the attractoin effect. Unpublished Paper.

Ok, E. A., P. Ortovela, and G. Riella (2015). Reveald (p)references theory. The American Economic Review 105, 299-321.

Pareto, V. (1906). Manuale di economia politica, Volume 13. Societa Editrice.

Plott, C. R. (1973). Path dependence, rationality, and social choice. Econometrica 41(6), 1075-1091.

Rubinstein, A. and Y. Salant (2006). A model of choice from lists. Theoretical Economics 1, $3-17$.

Salant, Y. and A. Rubinstein (2008). (A;f): Choice with frames. The Review of Economics 
Studies 75, 1287-1296.

Samuelson, P. A. (1938). A note on the pure theory of consumer's behavior. Economica 5(1), $61-71$.

Samuelson, W. and R. Zeckhauser (1988). Status quo bias in decison making. Journal of Risk and Uncertainty 1(1), 7-59.

Shafir, E., I. Simonson, and A. Tversky (1993). Reason-based choice. Cognition 49, 11-36.

Sims, C. A. (2003). Implications of ratioinal inattention. Journal of Monetary Economics 50, 665-690.

Teppan, E. C. and A. Felfering (2012). Minimization of decoy effects in recommneder result sets. Web Intellegence and Agent Systems: An International Journal 10, 385-395.

Tversky, A. and D. Kahneman (1991). Loss aversion in riskless choice: A reference-dependent model. The Quarterly Journal of Economics 106(4), 1039-1061.

Tversky, A. and I. Simonson (1993). Context-dependence preferences. Managemenet Science 39(10), 1179-1189. 